\title{
Melatonin-induced cold and drought tolerance requires brassinosteroids and hydrogen peroxide signaling in perennial ryegrass
}

Juanjuan Fu ( $\square$ fujuanjuan@nwafu.edu.cn )

Northwest Agriculture and Forestry University

Shentong Zhang

Huina Jiang

Xinfei Zhang

Hao Gao

Peizhi Yang

Tianming Hu

\section{Research Article}

Keywords: Brassinosteroids, cold stress, drought stress, Lolium perenne, melatonin, transcription regulation

Posted Date: September 2nd, 2021

DOI: https://doi.org/10.21203/rs.3.rs-867265/v1

License: (9) This work is licensed under a Creative Commons Attribution 4.0 International License. Read Full License 


\section{Abstract}

Aims The interplay between melatonin and brassinosteroids in enhancing tolerance to cold and drought and the underlying molecular mechanisms of this relationship still remain unknown.

Methods Combined physiological and transcriptomic analyses were used to clarify the crosstalk of melatonin and brassinosteroids in perennial ryegrass. Brassinosteroids biosynthesis inhibitor propiconazole, Arabidopsis brassinosteroids-receptor mutant bri1-9, bak1, and $\mathrm{H}_{2} \mathrm{O}_{2}$ deficient rbohC and rbohF mutants were used to analyzed the role of $\mathrm{H}_{2} \mathrm{O}_{2}$ in melatonin and brassinosteroids-mediated tolerance to the stress.

Results Melatonin-enhanced cold and drought tolerance of perennial ryegrass depends on the duration of dose and stress that were applied. Melatonin activates the expression of NADPH oxidase-related genes to promote $\mathrm{H}_{2} \mathrm{O}_{2}$ production when exposed to short-term cold/drought stresses but reduced accumulation of $\mathrm{H}_{2} \mathrm{O}_{2}$ under long-term stress. This was associated with an increase in enzymatic and non-enzymatic antioxidant activities by regulation of their gene expression and modulation of the ascorbate-glutathione cycle. Moreover, exogenous melatonin increased the biosynthesis of melatonin and brassinosteroids or the expression of signaling-related genes. However, the effects of melatonin on the expression of BR biosynthesis and signaling-related genes were inhibited in the $r b o h C$ and $r b o h F$ mutants. Chemical scavenging of $\mathrm{H}_{2} \mathrm{O}_{2}$ attenuated melatonin-mediated growth in the Arabidopsis wild-type, but the bak1 and bri1.9 mutants were relatively insensitive. Consistently, Inhibition of $\mathrm{H}_{2} \mathrm{O}_{2}$ production impaired the effect of melatonin and brassinosteroids on seed germination and the root growth of perennial ryegrass.

Conclusions This research reveals a novel regulatory mechanism of the crosstalk of $\mathrm{H}_{2} \mathrm{O}_{2}$ and brassinosteroid signaling in melatonin-induced cold/drought tolerance in perennial ryegrass.

\section{Introduction}

Perennial ryegrass (Lolium perenne L.), a cool-season turfgrass, is widely used for golf courses, parks and athletic fields because of its rapid establishment and attractive, leafy appearance in temperate regions throughout the world (Zhang et al. 2017). Cold and drought stress can adversely affect the turf quality of ryegrass or restrain its use in many areas. A decline in turf quality and canopy, leaf senescence and wilting under cold or drought stress are important concerns with the ryegrass turf management in a wide range of geographic locations (Sheikh Mohammadi et al. 2017). Genetic engineering and conventional breeding techniques to improve the tolerance to abiotic stresses are expensive, complicated, and time consuming or even unacceptable in many countries around the world (Moshelion et al. 2015). Priming using active small molecule substances is a rapidly emerging field in plant stress physiology and crop stress management, since it serves as an attractive alternate tool to enhance the tolerance of plants to abiotic stress (Arnao and Hernández-Ruiz et al. 2019; Sun et al. 2021; Tiwari et al. 2021). 
Melatonin (N-acetyl-5-methoxytryptamine) and brassinosteroids (BRs) are multifunctional phyotoprotectants that are involved in multiple physiological processes, such as promoting seed germination (Wang et al. 2018; Li et al. 2020), regulating plant growth and root morphology (Wei et al. 2015), alleviating the inhibition of photosynthesis (Fang et al. 2019), boosting osmoregulation, conducting primary and secondary metabolism (Planas-Riverola et al. 2019), and protecting against abiotic and/or biotic stresses (Campos et al. 2019; Sharma et al. 2020). The ability of melatonin to alleviate stress in plants is associated with its function as an antioxidant scavenging reactive oxygen/nitrogen species (ROS/RNS) (Sun et al. 2021). It is well established that the application of exogenous melatonin increases the activities of various antioxidant enzymes and the levels of ascorbic acid (AsA) and reduced glutathione (GSH) in turfgrass, such as perennial ryegrass, tall fescue, and bermudagrass, thereby reducing the accumulation of ROS and alleviating the oxidative damage induced by environmental stresses (Shi et al. 2015; Alam et al. 2018; Zhang et al. 2017) .

Brassinosteroid (BR) signaling mediates adaptation to stress via several mechanisms, including finetuning stress-responsive transcript networks (Ye et al. 2017; Lv et al. 2018), activating antioxidant defense (Xia et al. 2018), and promoting the production of osmoprotectants (Fàbregas et al. 2018). Several studies have also reported that BRs interact with hydrogen peroxide $\left(\mathrm{H}_{2} \mathrm{O}_{2}\right)$ and nitric oxide (NO) to induce stress tolerance (Lv et al. 2018; Planas-Riverola et al. 2019; Li et al. 2020). The application of exogenous $\mathrm{BR}$ increases the production of $\mathrm{H}_{2} \mathrm{O}_{2}$ in Arabidopsis seedling, and subsequent oxidative modifications mediated by $\mathrm{H}_{2} \mathrm{O}_{2}$ enhance the transcriptional activity of BZR1 (BRASSINAZOLERESISTANT 1), thus, contributing to the induction of plant development by BRs (Tian et al. 2018). Fang et al. (2019) also demonstrated that BRs act as positive regulators of photoprotection through BZR1, which directly activate the transcription of RESPIRATORY BURST OXIDASE HOMOLOG1 (RBOH1) and induce the production of $\mathrm{H}_{2} \mathrm{O}_{2}$ in response to chilling stress in tomato.

Furthermore, accumulating evidence suggests that melatonin also acts as an endogenous plant biostimulator or crosstalk with other signaling molecule, such as $\mathrm{H}_{2} \mathrm{O}_{2}$ mediates plant tolerance to abiotic stress through the regulation of genes involved in the DREB/CBF, ZAT, or CAMTA1-dependent pathways (Gong et al. 2017; Wang et al. 2018; Tiwari et al. 2020). Treatment with exogenous melatonin induced the expression of several BR biosynthetic genes, while the SNAT2 RNAi lines produced less BR than the wild type (Hwang and Back 2018). A similar study aslo demonstrated that melatonin regulates the growth of rice seedlings either with or without the biosynthesis of BRs (Lee and Back 2018). Interstingly, a study reported that lines of the melatonin-deficient mutants oscomt and ost5h in which the biosynthesis of BRs was decreased were tolerant to stress, whereas melatonin-deficient ostdc seedlings in which the levels of BRs did not decrease failed to exhibit increased stress tolerance (Hwang and Back 2019). Therefore, although a few research has been performed to decipher the functions of melatonin and BRs in plant morphogenesis and abiotic stress responses, more research is needed to unravel the precise mechanism of crosstalk between melatonin and BR signaling in response to cold and drought stress. In this study, we report that BR plays a critical role in the melatonin-mediated mitigation of cold and drought stress in perennial ryegrass by triggering antioxidant activities and enhancing photosynthetic capacities. 
Melatonin is induced by cold and drought stress, and it also acts as a signaling molecule that activates the expression of $\angle P R B O H B$ and $\angle P R B O H C$ genes and the subsequent accumulation of $\mathrm{H}_{2} \mathrm{O}_{2}$. This interaction with $\mathrm{BR}$ signaling participates in the melatonin-induced tolerance to cold and drought in perennial ryegrass.

\section{Materials And Methods}

\section{Plant materials and stress treatments}

Perennial ryegrass (cv. 'Pinnacle III') seeds used in this study were obtained from Beijing RYTWAY Seed Co., LTD (Beijing, China). After surface sterilization with $2.5 \% \mathrm{NaClO}$, the healthy seeds were sown in plastic pots (10 cm in diameter and $40 \mathrm{~cm}$ in depth) filled with vermiculite. The germinated seedlings were thinned to 30 uniform seedlings in each pot. The plants were grown in growth chambers with a temperature of $25 / 20^{\circ} \mathrm{C}$ (day/night), a $16 / 8 \mathrm{~h}$ light/dark cycle, and photosynthetic photon flux density of $350 \mu \mathrm{mol} \mathrm{m} \mathrm{m}^{-2} \mathrm{~s}^{-1}$. The seedlings were watered with Hoagland's nutrient solution every $3 \mathrm{~d}$. To study the effects of exogenous MET, 4-week-old seedlings were sprayed with different concentrations $(0,20,100$, and 1,000 $\mu \mathrm{M}$ ) of MET for $7 \mathrm{~d}$ before exposure to cold/ drought stress. The plants were exposed to cold stress by transfer to a growth chamber maintained at $4^{\circ} \mathrm{C}$ using the photoperiodic conditions described above. Drought stress was imposed by withholding watering. The plants treated with chemicals were deprived of water for 5 and $10 \mathrm{~d}$. For cold and drought tolerance experiments, the 4-week-old seedlings were sprayed with deionized water or MET $(100 \mu \mathrm{M})$, a BRs donor (2,4-epibrassinoide [EBL, $0.1 \mu \mathrm{M}])$, and MET plus the BR biosynthesis inhibitor propiconazole PPZ $(2 \mu \mathrm{M})$ for $7 \mathrm{~d}$ before exposure to cold or drought stress. Melatonin, EBL, and PPZ (Sigma-Aldrich, St. Louis, MO, USA) were dissolved in ethanol followed by dilution with distilled water at a ratio of 1/10,000 (v/v). For RNA-Seq assays, 28-day-old seedlings were sprayed with deionized water or MET $(100 \mu \mathrm{M})$ and then transferred to cold $\left(4^{\circ} \mathrm{C}\right)$ for $3 \mathrm{~d}$ and drought for $2 \mathrm{~h}$. The leaves were put on a filter paper and kept in dry air for short-term drought treatment. For germination assays, 50 healthy sterilized perennial ryegrass seeds were sown on $1 / 2 \mathrm{MS}$ media with different concentration of MET, EBL, MET or EBL plus dimethylthiourea (DMTU) exposed to $4^{\circ} \mathrm{C}$ or $250 \mathrm{mM}$ mannitol treatment. The seeds were germinated in the dark on $1 / 2 \mathrm{MS}$ medium as the control. Germination was defined as the emergence of radicles. DMTU was purchased from Solarbio Life Science (Beijing, China). To determine the root phenotpype of perennial ryegrass, 21-d-old seedlings grown in hydroponic culture were treated with $100 \mu \mathrm{M}$ MET or MET plus $0.5 \mathrm{mM}$ DMTU under cold or 250 $\mathrm{mM}$ mannitol treatment for $7 \mathrm{~d}$. Root length, surface area, and volume were determined using a LA-S root system scanner (Wseen, Shenzheng, China). The A. thaliana BR-receptor mutant bri1-9, the co-receptor mutant bak $1, \mathrm{H}_{2} \mathrm{O}_{2}$ deficient $r b o h C$, and $r b o h F$ mutants used in this study were in the Columbia- 0 background. For plant growth of $A$. thaliana wild type (WT), bri1-9 and bak1 mutants, 7-day-old seedlings with uniform growth were transferred onto $1 / 2 \mathrm{MS}$ agar plates pretreated with or without $50 \mu \mathrm{M}$ MET and MET plus $0.5 \mathrm{mM}$ DMTU exposure to cold for $14 \mathrm{~d}$ and $150 \mathrm{mM}$ mannitol for $7 \mathrm{~d}$, respectively.

\section{Quantitative RNA-Seq data analysis}


RNA-Seq experiments were performed using Illumina Novaseq ${ }^{\text {TM }} 6000$ instrument at the Lc. Biotech Co., Ltd. (Hangzhou, China, http://www.lc-bio.com/). according to the manufacturer's procedure. De novo assembly of the transcriptome was performed with Trinity 2.4.0 (Grabherr et al. 2011). Differentially expressed genes (DEGs) analysis by calculating the fragments per kilobase per million reads. The threshold of significantly differential expression was set to $p<0.05$ and $\mid \log 2$ (fold change) $\mid \geq 1$ by $R$ package edgeR (Robinson et al. 2010). GO and KEGG enrichment analysis were performed to explore the function and biological pathways in which the DEGs were involved.

\section{Quantitative real-time PCR (RT-qPCR) analysis}

Total RNA was extracted using TRIzol reagent. RT-qPCR analysis using a LightCycler 480 RTPCR system (Roche Applied Science, Germany). The specific primers used for expression analysis are listed in Table S1. The relative expression levels of genes were calculated using the $2^{-\triangle \triangle C T}$ method.

\section{Assays of $\mathrm{H}_{2} \mathrm{O}_{2}$ and relative electrolyte leakage (REL)}

We used DAB (3,3'-diaminobenzidine) staining to detect $\mathrm{H}_{2} \mathrm{O}_{2}$ producing, as described our previous study (Fu et al. 2019). The hydrogen peroxide detection kit (Solarbio, Beijing) was used to $\mathrm{H}_{2} \mathrm{O}_{2}$ quantification by recording absorbance at $530 \mathrm{~nm}$. The REL was determined and calculated as described by Miura et al. (2007) .

\section{Assays of antioxidant enzyme activities and antioxidant concentrations}

Fresh leaf samples were used for enzyme extraction. The extraction and measurement of CAT, POD, APX, and GR using $50 \mathrm{mM}$ phosphate buffer $(\mathrm{pH} 7.8)$ containing $1 \mathrm{mM}$ EDTA and $2 \%$ PVP at $4^{\circ} \mathrm{C}$, as described by previously (Xia et al. 2020). GST activity was determined at $340 \mathrm{~nm}$ according to the protocol as described by Hossain et al. (2010). The amounts of reduced (GSH) and oxidized (GSSG) glutathione were detected at $412 \mathrm{~nm}$, and the content of reduced (AsA) and oxidized (DHA) ascorbate were estimated at $265 \mathrm{~nm}$ according to Solarbio assay kit, respectively.

Measurement of net photosynthetic rate $\left(P_{n}\right)$, maximum quantum efficiency of PSII $\left(F_{v} / F_{m}\right)$ and chlorophyll (Chl) content

Photosynthetic rate was measured using a Li-6400 Portable Photosynthesis System (Li-Cor, Lincoln, NE, USA). The $F_{v} / F_{m}$ was measured using an closed-ended chlorophyll fluorescence imaging (PSI, Czech, Germany). The extraction of $\mathrm{Chl}$ using $80 \%$ acetone as previously described (Fu et al. 2016). The supernatant was used to determine the absorbance spectrophotometrically at 645 and $663 \mathrm{~nm}$ against $80 \%$ acetone as blank.

\section{Determination of melatonin and brassinosteroids}

The endogenous brassinosteroids were quantified by enzyme-linked immunosorbent assay using Plant Brassinosteroids ELISA kit (F4963-B; Shanghai Fanke Co., Ltd., Shanghai China), as described in our 
previous study (Fu et al. 2019). Melatonin was extracted and assayed using a Plant Melatonin ELISA Kit (R\&D Systems, Minneapolis, MN, USA) as previously described (Fu et al. 2017).

\section{Statistical analysis}

The experiment was conducted in a completely randomized design with three independent biological replicates. For statistical analysis, the data were analyzed using an analysis of variance (ANOVA) using SPSS 22.0 software, and $P<0.05$ were considered statistically significant according to a Duncan's test.

\section{Results}

\section{Exogenous melatonin improved cold and drought tolerance in perennial ryegrass}

The exogenous application of melatonin reduced the inhibition of plant growth under both cold and drought stress in a dose and stress treatment duration-dependent manner, while no significant differences were observed in non-treated and plants pre-treated with different concentrations of melatonin under normal conditions (Fig. 1). Pretreatment with 20, 100 and 1,000 $\mu \mathrm{M}$ melatonin resulted in a higher fresh weight and lower REL when the plants were exposed to $3 \mathrm{~d}$ of cold stress. Treatment with $100 \mu \mathrm{M}$ was the most effective. Along with the extension of cold stress, the pre-treatment of plants with a higher concentration of mealtonin $(100$ and $1,000 \mu \mathrm{M})$ grew better, whereas a lower fresh weight and higher REL were found in the plants treated with $20 \mu \mathrm{M}$ of melatonin (Figs. 1a, c). A similar changing patterns of fresh weight and REL was observed in the melatonin-treated plants under drought conditions (Figs. 1b, d). As expected, $100 \mu \mathrm{M}$ melatonin significantly alleviated the stress damage caused by cold and drought treatment, as indicated by less severe phenotypes (Fig. 1e).

\section{Systematic transcriptome shifts modulation by melatonin in perennial ryegrass}

Treatment with melatonin causes a dramatic change in gene expression in perennial ryegrass under cold and drought stress. In total, 11,588 differentially expressed genes (DEGs) were identified in at least one sample of plants subjected to cold and drought or treatment with melatonin compared with their respective controls. Among these, a number of 5,279 (2,848 up- and 2,431 down-regulated), 5,105 (2,307 up- and 2,798 down-regulated), 2,249 (727 up- and 1,522 down-regulated), and 7,162 (3,943 up- and 3,218 down-regulated) DEGs were obtained in cold, drought, cold plus melatonin, and drought plus melatonin treated seedlings, respectively (Supplementary Fig. S1a, b).

A GO enrichment analysis of the DEGs showed that $347 \mathrm{GO}$ terms were notably enriched in at least one of the treatments (FDR $<0.05$; Supplementary Table S2). An overrepresentation of genes in the Go biological processes related to abiotic stress responses, redox and osmotic homeostasis (i.e., AsA-GSH cycle, glutathione, polyamine and proline metabolism), regulation of phytohormones (i.e., ABA, brassinosteroid, 
melatonin, ethylene, jasmonic acid, and salicylic acid), photosynthesis (i.e., chloroplast organization, chlorophyll biosynthesis, and photosystem I/II assembly), carbohydrate metabolism (i.e., trehalose, galactose, raffinose, maltose, and sucrose), lipid metabolism (i.e. sterol, phospholipid, and wax), and the phenylpropanoid pathway, i.e., flavonoids. Moreover, signal transduction (i.e., MAPK signaling, phosphorylation, and ubiquitination), protein folding, translation and transcriptional regulation were closely related to the response of melatonin-mediated cold and drought stress (Supplementary Table S2). Several transcription factors, including DREB1A, ERF1, ERF109, MYB108, MYB4, WRKY30, WRKY53, bZIP73, ZAT6, and MADS57, were induced by melatonin under cold and drought conditions. Interestingly, the genes that encode dehydrin, such as DHN1, DHN3, RAB15, CS66, COR47, and COR413PM1, were upregulated by melatonin under abiotic stress (Supplementary Table S3). Nineteen differentially expressed transcripts from RNA-Seq were used to validate the RNA-Seq data using qPCR analysis. The qPCR measurements were highly correlated with the transcript abundance from RNA-Seq analysis ( $y=$ $0.9368 x+0.1062, r=0.8992)$ (Supplementary Fig. S1c).

The significantly enriched KEGG pathways included brassinosteroid biosynthesis, tryptophan metabolism, phenylalanine metabolism, biosynthesis of unsaturated fatty acids, alpha-linolenic acid metabolism, glutathione metabolism, starch and sucrose metabolism, galactose metabolism, phenylpropanoid biosynthesis, flavonoid biosynthesis, MAPK signaling, and ubiquitin mediated proteolysis. Additionally, melatonin changed the citrate cycle and glycolysis/gluconeogenesis processes (Supplementary Table S2).

\section{ROS producing and scavenging processes are mediated by melatonin}

A total of 43 genes related to production of $\mathrm{H}_{2} \mathrm{O}_{2}$ were substantially activated under cold and drought stress, including $R B O H B, R B O H C$, and DGS1 (Supplementary Fig. S2). Melatonin induced the expression of genes for $R B O H B, R B O H C$, and $D G S 1$ following exposure to short-term cold and drought stress but reduced their levels of expression and accumulation of $\mathrm{H}_{2} \mathrm{O}_{2}$ under long-term stress (Fig. 2). Notably, the upregulation of melatonin genes was related to the mitochondrial respiratory chain complex and chloroplast respiratory chain (Supplementary Fig. S2). Polyamine oxidase-related genes, which catalyze the degradation of polyamines to induce the accumulation of $\mathrm{H}_{2} \mathrm{O}_{2}$, i.e., PAO2, PAO4, and PAO5 were induced by cold or drought stress but repressed by treatment with melatonin. The genes that encode enzymes involved in spermine and proline biosynthesis were activated by melatonin, including SPMS, SAMDC, SPDSYN1, P5CS, and P5CR (Supplementary Fig. S2).

The AsA-GSH cycle and glutathione metabolism also participate in melatonin-mediated cold and drought tolerance. A total of 51 genes were involved in glutathione metabolism, including glutathione Stransferases (GSTs) and glutaredoxin that were markedly induced by melatonin under cold and drought stress. Seventeen up-regulated genes related to the AsA-GSH cycle, including MDAR2, MDAR5, APX7, and $A P X 8$, were found in plants that were treated with melatonin. Superoxide dismutase, peroxidases, catalase, and thioredoxin, as well as ferredoxin, were also differentially regulated by melatonin 
(Supplementary Fig. S3). The physiological experiments also revealed that melatonin significantly increased the ratio of AsA/DHA and GSH/GSSG and enhanced the activities of APX, GR, GST, and CAT following exposure to $5 \mathrm{~d}$ of cold and $10 \mathrm{~d}$ of drought stresses (Supplementary Fig. S4).

\section{Brassinosteroid biosynthesis and the signaling pathway are induced by melatonin}

Many hormone-related genes were differentially regulated by melatonin in perennial ryegrass and were assigned to nine major categories of phytohormones (Supplementary Table S3). Among them, 10 genes that encod the biosynthessis of melatonin were significantly induced by melatonin. More importantly, 37 genes related to the biosynthesis, response, and signal transduction of brassinosteroid were substantially induced by melatonin, particularly SERK1 and IWS1, the genes involved in brassinosteroid-mediated signaling transduction, and CYP750A1, CYP707A5, CYP707A7, CYP87A3, and CYP90D2, the genes involved in biosynthesis of brassinosteroid (Figs. 3a-k). The expression of ASMT1 gene that encodes the key biosynthetic enzyme in melatonin biosynthesis was upregulated after $1 \mathrm{~h}$ of cold or drought stress. The expression of OMT2 and ASMT3 under drought and cold stress was continually higher than that of the control. All three genes, $A S M T 1, A S M T 3$, and $O M T 2$, were gradually induced by melatonin and reached a maximum at 3 or $6 \mathrm{~h}$ following the treatment of leaves with cold or drought. The level of expression of CYP87A3, CYP707A7, and CYP750A1, continuously increased when subjected to stress and reached their maximal levels at $6 \mathrm{~h}$ of cold and drought, respectively. CYP90D2 and CYP707A5 were more highly expressed following 1 and $3 \mathrm{~h}$ of drought stress, respectively. Cold stress also induced the expression of CYP707A5 and CYP90D2 genes with a higher expression at $1 \mathrm{~h}$. Pretreatment with melatonin significantly enhanced the transcription levels of the CYP90D2, CYP707A5, and CYP707A7 genes during the whole period of stress, while a slight change was observed in the level of expression of CYP87A3 and CYP750A1 genes at 1 and $3 \mathrm{~h}$ of cold or drought stress compared with nontreated stressed plants, respectively. Additionally, IWS1 and SERK1 were up-regulated by melatonin following exposure to cold or drought stress (Figs. 3e-k). As expected, cold and drought stress increased the content of melatonin in leaves after $1 \mathrm{~h}$ with leaf brassinosteroids that showed a similar tendency. Melatonin pretreatment improved the levels of endogenous melatonin and BRs (Figs. 3i, m).

BRs play a partial role in the improvement of cold and drought tolerance of perennial ryegrass by melatonin

Our GO and KEGG enrichment analysis showed that a total of 147 genes involved in photosynthesis were identified after melatonin treatments (Supplementary Fig. S5). The expression of seven genes that encode the assembly of photosystem II (PS II), including PsbM, PsbE, PsbB, PsbA, LTO1, SEP1 and EGY1, were induced by melatonin. Melatonin also markedly induced the expression of genes that encode the PSIl-associated light-harvesting complex and oxygen evolving complex, i.e. PSBR, PSBS, PSBO, PPD3, and $P N S B 5$. Genes related to the chlorophyll a-b binding protein involved in light harvesting in photosystem I were upregulated by melatonin treatment following exposure to cold and drought stress. These genes included eight $W H A B 1.6$, six $C A B 2 R$, three $C A B 3 A$, four $C A B 2 R$, two $C A B 2$, two $\angle H B C$. Exogenous 
melatonin increased the content of chlorophyll in the leaves of perennial ryegrass exposed to cold and drought stress, which was consistent with the upregulation of genes that encode chlorophyll biosynthesis and the downregulation of the genes involved in the catabolism of chlorophyll (Fig. 4; Supplementary Fig. S5). Furthermore, the genes involved in PSII repair were increased by melatonin. To confirm this, we measured $F_{v} / F_{m}, P_{n}$, and $\mathrm{Chl}$ content. Plants treated with melatonin or EBL had a higher $F_{v} / F_{m}$ and lower REL and accumulation of $\mathrm{H}_{2} \mathrm{O}_{2}$ after $5 \mathrm{~d}$ of cold or $10 \mathrm{~d}$ of drought stress. However, pretreatment with PPZ substantially inhibited the induction of cold or drought tolerance by melatonin as indicated by a lower $F_{v} / F_{m}$ and higher REL and accumulation of $\mathrm{H}_{2} \mathrm{O}_{2}$ (Figs. 4a-d). Decreased activitives of CAT, POD, APX, GR, and GST and a lower ratio of AsA/DHA and GSH/GSSG were observed in plants treated with melatonin plus PPZ (Supplementary Fig. S4).

To further confirm whether BR signaling was involved in the mediation of cold and drought tolerance by melatonin, we analyzed the effects of exogenous melatonin on morphologies in A. thaliana bri1.9 and bak1 mutants. The bri1.9 and bak1 mutants were more sensitive to cold and drought stress, and pretreatment with melatonin could alleviate the damage caused by stress in the WT and bak1 plants but exhibited no obvious improvement in the bri1.9 mutants (Fig. 5a).

Consistent with the phenotypic analysis, a stronger DAB staining density of $\mathrm{H}_{2} \mathrm{O}_{2}$ was detected in the leaves of bak1 mutants exposed to cold and drought stress compared with the WT plants (Fig. 5b). Pretreatment with melatonin decreased $\mathrm{H}_{2} \mathrm{O}_{2}$ accumulation in both genetypes under cold or drought stress, while the accumulation of $\mathrm{H}_{2} \mathrm{O}_{2}$ in the bak1 mutants was maintained at a high level. This indicates that signaling by BRs plays key role in the regulation of cold and drought tolerance coupled with $\mathrm{H}_{2} \mathrm{O}_{2}$ levels regulated by melatonin.

\section{An appropriate accumulation of $\mathrm{H}_{2} \mathrm{O}_{2}$ is required for melatonin- and BRs-induced plant growth}

To determine the important role of $\mathrm{H}_{2} \mathrm{O}_{2}$ signaling in the mediation of responses to cold and drought stress by melatonin in perennial ryegrass. We utilized an $\mathrm{H}_{2} \mathrm{O}_{2}$ scavenger DMTU to analyze the effects of melatonin and BRs on seed germination and seedling growth under cold and drought stress. Both cold and drought stress lowered the rate of seed germination of perennial ryegrass, while the application of $100 \mu \mathrm{M}$ melatonin and $0.1 \mu \mathrm{M}$ BRs significantly improved seed germination under the stresses but delayed seed germination at a higher concentration $(1,000 \mu \mathrm{M}$ melatonin and $1 \mu \mathrm{M}$ BRs). Interestingly, the plants pretreated with melatonin and DMTU ( 0.5 and $5 \mathrm{mM}$, respectively) exhibited a lower rate of seed germination than those treated with melatonin exposed to both cold and drought stress. A lower concentration of DMTU consistently led to an increase in the germination rate when the seeds were treated with BRs under cold and drought stress compared with treatment with BRs alone, while the germination of seeds pretreated with BRs and a higher dose of DMTU level was inhibited (Fig. 6; Supplementary Fig. S6). 
The application of melatonin alleviated the inhibition of roots induced by cold or drought (Fig. 7a). In plants pretreated with melatonin, the root length, surface area, and volume were $111.32 \%, 68.12 \%, 30.58 \%$ and $65.64 \%, 52.59 \%, 17.24 \%(P<0.05)$ higher after exposure to drought and cold stress compared with non-melatonin treated plants, respectively. Pretreatment with DMTU and melatonin inhibited the effect of melatonin on the growth of perennial ryegrass seedling roots under cold and drought stress (Figs. $7 \mathrm{~b}-\mathrm{d}$ ).

Interaction with $\mathrm{BR}$ and $\mathrm{RBOH}$-mediated $\mathrm{H}_{2} \mathrm{O}_{2}$ signaling plays a critical role in melatonin-induced plant growth and stress tolerance

To further clarify the interactive role of $\mathrm{H}_{2} \mathrm{O}_{2}$ and $\mathrm{BR}$ signalings in the regulation of cold and drought responses by melatonin, the growth phenotypes of the WT and bak 1 and bri1.9 mutants were analyzed following treatment with DMTU and melatonin. We found that the appication of DMTU and melatonin inhibited the growth of WT, bak1 and bri1.9 mutants under cold and drought stress compared with plants treated with melatonin, but the bak1 and bri1.9 mutants were only slightly sensitive (Fig. 5a).

Moreover, the effects of melatonin on the expression of BR biosynthesis and signaling-related genes, $D W F 4, B Z R 1, B R / 1$ were inhibited in the rbohC and $r b o h F$ mutants. Melatonin and BRs induced the expression of stress-responsive genes in WT, but this induction was impaired in the rbohC and rbohF mutants. Pretreatment with $\mathrm{H}_{2} \mathrm{O}_{2}$ effectively restored the expression of these stress-related genes in rbohC and rbohF mutants (Fig. 8).

\section{Discussion}

\section{BRs involved in the melatonin-mediated antioxidant defense capacity in perennial ryegrass}

Melatonin acts as an antioxidant and important signal molecule that can trigger the entire antioxidant system in turfgrass plants to protect cells from the oxidative stress induced by abiotic stress, such as cold and drought (Alam et al., 2018; Zhang et al. 2017; Sun et al. 2021; Yao et al. 2021). Similarly, it has been reported that BRs alleviate oxidative damages induced by cold in tomato using a signaling cascade that involves RBOH1, glutaredoxin, and 2-cysteine peroxiredoxin (Xia et al. 2018). In this study, we reported that the regulation of tolerance to cold and drought tolerance by melatonin has a bimodal concentration dependence in perennial ryegrass (Fig. 1), which is consistent with research by Gong et al. (2017), who found that $100 \mu \mathrm{M}$ melatonin was more effective. The application of $100 \mu \mathrm{M}$ melatonin enhanced the activities of APX, GR, GST, and CAT and the ratio of AsA/DHA and GSH/GSSG under cold and drought stress, while the inhibitor (PPZ) of BRs biosynthesis partially reduced the defense responses induced by melatonin (Supplementary Fig. S4), indicating that BRs have a crucial role in the signaling pathway in the cold and drought stress response of perennial ryegrass that is mediated by melatonin. This result was supported by a recent study which demonstrate that melatonin decreases the levels of methylation of the antioxidant genes $A P X 2, P O D 52$ and GST, which enables radish plants to scavenge lead-induced ROS and decrease oxidative stress (Tang et al. 2021). A similar study showed that the 
melatonin biosynthetic genes TDC2, ASMT2 interact with APX2 in cassava (Bai et al. 2020), which highlights the importance of melatonin in the regulation of redox homoeostasis in plants.

The biosynthesis of melatonin primarily occurs in mitochondria and chloroplasts in plants, which is closely related to its antioxidant functions (Wang et al. 2017; Zheng et al. 2017). The present study showed that melatonin upregulated the expression of genes related to the mitochondrial respiratory chain complex and improved photosynthetic efficiency via upregulated expression of PS II assembly, lightharvesting-related genes and the downregulation expression of genes related to catabolism of chlorophyll, thus, maintaining higer $P_{n}, F_{v} / F_{m}$, and lower $\mathrm{Chl}$ degradation and levels of $\mathrm{H}_{2} \mathrm{O}_{2}$ under cold and drought stress. Consistently, a study regards to melatonin improving photosynthetic efficiency has been reported in Coffea arabica (Campos et al. 2019). Treatment with PPZ attenuated the repair of photosynthesis and the detoxification of $\mathrm{H}_{2} \mathrm{O}_{2}$ mediated by melatonin (Figs. 4; Supplementary Fig. S3), which could be confirmed by the findings reported by Fang et al. (2019) who demonstrated that BRs act as a positive regulator of photoprotection in a RBOH1-PROTON GRADIENT REGULATION5-dependent manner in response to chilling stress. Thus, these data suggest that BRs participate in the melatoninmediated redox pathway in the cold and drought stress response of perennial ryegrass.

\section{Brassinosteroid biosynthesis and its signaling pathway are induced by melatonin}

A few researches demonstrated that melatonin and BRs function synergistically to regulate plant morphogenesis (Hwang and Back 2018; Lee and Back 2019). However, there are still contrasting views about crosstalk of melatonin and BRs signaling in response to abiotic stress (Hwang and Back 2019). Our data showed that melatonin improves both endogenous melatonin and the content of BRs under cold and drought stress (Fig. 3). It is likely that melatonin self-regulates the expression of genes of its biosynthesis when the plant is subjected to stress (Arnao and Hernández-Ruiz 2019). Furthermore, melatonin also increased the level of transcription of genes related to BRs signaling in perennial ryegrass (Fig. 3), but failed to alleviate the stresses-caused damage in the bri1.9 mutants (Fig. 5), indicating that $\mathrm{BR}$ might be a downstream signaling of melatonin in response to environmental stress. The mechanism that underlies the melatonin/BRs-mediated signaling pathway in plant abiotic stress responses is associated with its modulation of relevant transcription factors (Planas-Riverola et al. 2019; Tiwari et al. 2021). For example, under cold and drought stress, melatonin upregulated the expression of DREBs, CAMTA1, ZAT6, ZAT10, and ZAT12 (Shi et al. 2015; Wang et al. 2018). Additionally, the BRs-regulated bHLH transcription factors CESTA and BZR1 stimulate the transcription of CBFs genes, which activate the expression of cold responsive genes (Eremina et al. 2016; Li et al. 2017). Consistent with the findings of these studies, our data showed that exogenous melatonin upregulated the expression of DREB1A, ERF1, ERF109, MYB108, MYB4, WRKY30, WRKY53, bZIP73, ZAT6, and MADS57 and several genes that encod dehydrin (Supplementary Table S3); these genes have been reported to be involved in abiotic stress, including cold and drought stress (Shi et al. 2015; Xu et al. 2019; Liu et al. 2019; Wang et al. 2019; $\mathrm{Hu}$ et al. 2020). These results suggest that melatonin and BRs function together in response to cold and 
drought stress, in which melatonin induces the accumulation of BRs and subsequently activates a wide range of stress-responsive genes.

\section{$\mathrm{H}_{2} \mathrm{O}_{2}$ signaling contributes to melatonin- and BRs-induced cold and drought tolerance}

Evidence has shown that melatonin induces the accumulation of $\mathrm{H}_{2} \mathrm{O}_{2}$ in plants, and, as a key signaling molecule, $\mathrm{H}_{2} \mathrm{O}_{2}$ mediates melatonin-induced seed germination, root growth, and tolerance to environmental stress (Chen et al. 2018; Wei et al. 2018; Arnao and Hernández-Ruiz 2019). Indeed, we found that melatonin upregulated the expression of $\angle p R B O H B$ and $\angle p R B O H C$ genes, accompanied by an increase in the production of $\mathrm{H}_{2} \mathrm{O}_{2}$ under short-term cold and drought stress (Fig. 2; Supplementary Fig. S2). This supported by a previous study that showed the initial $\mathrm{H}_{2} \mathrm{O}_{2}$ burst is followed by a burst of melatonin, which further triggers downstream signaling to respond to diverse stress (Gong et al. 2017; Li et al. 2021). Chemical scavenging of $\mathrm{H}_{2} \mathrm{O}_{2}$ partially blocked the induction of seed germination and root growth induced by melatonin or BRs following exposure to cold and drought stress (Figs. 6, 7).

Consistently, the previous study demonstrated that melatonin and $\mathrm{BRs}$-induced $\mathrm{H}_{2} \mathrm{O}_{2}$ production through RBOH dependent pathway contributes to an adaptive cellular mechanism that stimulates stress response genes and inhibits ROS bursts to protect plants against stress condition (Gong et al. 2017; Xia et al. 2018). This study demonstrated that melatonin-induced BR levels and signaling were attenuated in the rhohC and rbohF mutants, and maintained a higher level of accumulation of $\mathrm{H}_{2} \mathrm{O}_{2}$ in the bak 1 mutant in the presence of melatonin, indicating that the key role of crosstalk of BRs and $\mathrm{H}_{2} \mathrm{O}_{2}$ signaling in cold and drought tolerance regulated by melatonin. Furthermore, the scavenging of $\mathrm{H}_{2} \mathrm{O}_{2}$ compromised the growth of WT and the bak1 and bri1.9 mutants regulated by melatonin under cold and drought stress, but the two mutants were slightly sensitive. Additionally, both the rbohC and rbohF mutants showed the decreased expression levels induced by melatonin in the stress-related genes compared with the wild-type plants (Figs. 5, 8). These findings suggest that melatonin and BRs interdependently regulate cold and drought tolerance, and the crucial role of $\mathrm{H}_{2} \mathrm{O}_{2}$ in the interaction between melatonin and BRs that incudes tolerance to stress merits further study.

\section{Conclusions}

In summary, the present study revealed for the first time that the activated melatonin-BRs- $\mathrm{H}_{2} \mathrm{O}_{2}$ signaling networks contribute to regulate redox homeostasis and subsequently enhance tolerance to cold and drought in the turfgrass plants, a proposed model is presented in Fig. 9. Melatonin increases the levels of endogenous melatonin and the induction of BRs biosynthesis and signaling pathway in the plants exposed to initial phases of stress. In this process, the NADPH oxidase-dependent $\mathrm{H}_{2} \mathrm{O}_{2}$ signaling might acts as a downstream component, and this signaling network further activates relevant transcription factors and stress-related genes, such as DREB1A, ERF1, ERF109, MYB108, MYB4, WRKY30, WRKY53, bZIP73, ZAT6, MADS57, and several genes encode dehydrin, that have been reported to be involved in abiotic stress (Liu et al. 2019; Wang et al. 2019; Hu et al. 2020). 


\section{Declarations}

Acknowledgements

This work was supported by the National Natural Science Foundation of China (31901382, 31872411), the Fundamental Research Funds for the Central Universities (2452020161), and the Key Research and Development Program of Shaanxi Province (2021NY-007).

\section{Availability of data and material}

The raw RNA-Seq reads have been deposited in the National Center for Biotechnology Information BioProject database (http://www.ncbi.nlm.nih.gov/bioproject) with ID PRJNA747746.

\section{Conflict of interest}

The authors declare no competing interests.

\section{References}

1. Alam MN, Zhang LH, Yang L, Islam MR, Liu Y, Luo H, Yang PF, Wang QF, Chan ZL (2018) Transcriptomic profiling of tall fescue in response to heat stress and improved thermotolerance by melatonin and 24-epibrassinolide. BMC Genomics 19:224. http://doi.10.1186/s12864-018-4588-y

2. Arnao MB, Hernández-Ruiz J (2019) Melatonin: a new plant hormone and/or a plant master regulator?. Trends Plant Sci 24:38-48. http:// doi.10.1016/j.tplants.2018.10.010

3. Bai YJ, Guo JR, Reiter RJ, Wei YX, Shi HT (2020) Melatonin synthesis enzymes interact with ascorbate peroxidase to protect against oxidative stress in cassava. J Exp Bot 71:5645-5655. http://doi.10.1093/jxb/eraa267

4. Camposa CN, Ávilab RG, de Souzab KRD, Azevedob LM, Alves JD (2019) Melatonin reduces oxidative stress and promotes drought tolerance in young Coffea arabica L. plants. Agr Water Manage 211:37-47. http://doi.10.1016/j.agwat.2018.09.025

5. Chen Z, Gu Q, Yu X, Huang L, Xu S, Wang R, Shen W, Shen W (2018) Hydrogen peroxide acts downstream of melatonin to induce lateral root formation. Ann Bot 121:1127-1136. http://doi.10.1093/aob/mcx207

6. Eremina M, Unterholzner SJ, Rathnayake Al, Castellanos M, Khana M, Kugler KG, May ST, Mayer KFX, Rozhon W, Poppenberger B (2016) Brassinosteroids participate in the control of basal and acquired freezing tolerance of plants. P Natl Acad Sci USA 113:E5982-E5991. http://doi.10.1073/pnas.1611477113

7. Fabregas N, Lozano-Elena F, Blasco-Escámez D, Tohge T, Martínez-Andújar C, Albacete A, Osorio S, Osorio S, Bustamante M, Riechmann JL, Nomura T, Yokota T, Conesa A, Pérez Alfocea F, Fernie AR, Caño-Delgado Al (2018) Overexpression of the vascular brassinosteroid receptor BRL3 confers 
drought resistance without penalizing plant growth. Nat Commun 9:4680.

http://doi.10.1038/s41467-018-06861-3

8. Fang PP, Yan MY, Chi C, Wang MQ, Zhou YH, Zhou J, Shi K, Xia X, Foyer CH, Yu JQ (2019)

Brassinosteroids act as a positive regulator of photoprotection in response to chilling stress. Plant Physiol 180:2061-2076. http://doi.10.1104/pp.19.00088

9. Fu JJ, Sun PY, Luo YL, Zhou HY, Gao JZ, Zhao DH, Pubu ZM, Liu JL, Hu TM (2019) Brassinosteroids enhance cold tolerance in Elymus nutans via mediating redox homeostasis and proline biosynthesis. Environ Exp Bot 167:103831. http://doi.10.1016/j.envexpbot.2019.103831

10. Fu JJ, Gates RN, Xu YF, Hu TM (2016) Diffusion limitations and metabolic factors associated with inhibition and recovery of photosynthesis following cold stress in Elymus nutans Griseb. J Photoch Photobio B 163:30-39. http://doi.10.1016/j.jphotobiol.2016.08.008

11. Fu JJ, Wu Y, Miao Y, Xu Y, Zhao E, Wang J, Sun H, Liu Q, Xue Y, Xu Y (2017) Improved cold tolerance in Elymus nutans by exogenous application of melatonin may involve ABA-dependent and ABAindependent pathways. Sci Rep 7:39865. http://doi.10.1038/srep39865

12. Gong B, Yan YY, Wen D, Shi QH (2017) Hydrogen peroxide produced by NADPH oxidase: a novel downstream signaling pathway in melatonin-induced stress tolerance in Solanum lycopersicum. Physiol Plantarum 160:396-409. http://doi.10.1111/ppl.12581

13. Grabherr MG, Haas BJ, Yassour M, Levin JZ, Thompson DA, Amit I, Adiconis X, Fan L, Raychowdhury R, Zeng Q, Chen Z, Mauceli E, Hacohen N, Gnirke A, Rhind N, di Palma F, Birren BW, Nusbaum C, Lindblad-Toh K, Friedman N, Zeng Q (2011) Full-length transcriptome assembly from RNA-seq data without a reference genome. Nat Biotechno/29:644. http://doi.10.1038/nbt.1883

14. Hossain MA, Hasanuzzaman M, Fujita M (2010) Up-regulation of antioxidant and glyoxalase systems by exogenous glycinebetaine and proline in mung bean confer tolerance to cadmium stress. Physiol Mol Biol Pla 16:259-272. http://doi.10.1007/s12298-010-0028-4

15. Hu ZR, Huang XB, Amombo E, Liu A, Fan JB, Bi AY, Ji K, Xin H, Chen L, Fu JM (2020) The ethylene responsive factor CdERF1 from bermudagrass (Cynodon dactylon) positively regulates cold tolerance. Plant Sci 294:110432. http://doi.10.1016/j.plantsci.2020.110432

16. Hwang OJ, Back K (2018) Melatonin is involved in skotomorphogenesis by regulating brassinosteroid biosynthesis in rice plants. J Pineal Res 65:e12495. http://doi.10.1111/jpi.12495

17. Hwang OJ, Back K (2019) Melatonin deficiency confers tolerance to multiple abiotic stresses in rice via decreased brassinosteroid levels. Int J Mol Sci 20:5173. http://doi.10.3390/ijms20205173

18. Lee K, Back K (2019) Melatonin-deficient rice plants show a common semidwarf phenotype either dependent or independent of brassinosteroid biosynthesis. J Pineal Res 66:e12537. http:// doi.10.1111/jpi.12537

19. Li H, Guo YL, Lan ZX, Xu K, Chang JJ, Ahammed GJ, Ma J, Wei C, Zhang X (2021) Methyl jasmonate mediates melatonin-induced cold tolerance of grafted watermelon plants. Hortic Res 8:57. http://doi.10.1038/s41438-021-00496-0 
20. Li H, Ye K, Shi Y, Cheng J, Zhang X, Yang S (2017) BZR1 positively regulates freezing tolerance via cbf-dependent and cbf-independent pathways in Arabidopsis. Mol Plant 10:545-559. http://doi.10.1016/j.molp.2017.01.004

21. Li JG, Fan M, Hua W, Tian Y, Chen LG, Sun Y, Bai MY (2020) Brassinosteroid and hydrogen peroxide interdependently induce stomatal opening by promoting guard cell starch degradation. Plant Cell 32:984-999. http://doi.10.1105/tpc.19.00587

22. Liu CT, Schläppi MR, Mao BG, Wang W, Wang AJ, Chu CC (2019) The bZIP73 transcription factor controls rice cold tolerance at the reproductive stage. Plant Biotechnol J 17:1834-1849. http://doi.10.1111/pbi.13104

23. Lv BS, Tian HY, Zhang F, Liu JJ, Lu SC, Bai MY, Li CY, Ding ZJ (2018) Brassinosteroids regulate root growth by controlling reactive oxygen species homeostasis and dual effect on ethylene synthesis in Arabidopsis. PLoS Genet 14:e1007144. http://doi.10.1371/journal.pgen.1007144

24. Miura K, Jin JB, Lee J, Chan YY, Stirm V, Miura T, Ashworth EN, Bressan RA, Hasegawa YPM (2007) SIZ1-mediated sumoylation of ICE1 controls CBF3/DREB1A expression and freezing tolerance in Arabidopsis. Plant Cell 19:1403-1414. http://doi.10.1105/tpc.106.048397

25. Moshelion M, Altman A (2015) Current challenges and future perspectives of plant and agricultural biotechnology. Trends Biotechno/33:337-342. http://doi.10.1016/j.tibtech.2015.03.001

26. Planas-Riverola A, Gupta A, Betegon-Putze I, Bosch N, Ibanes M, Cano-Delgado Al (2019) Brassinosteroid signaling in plant development and adaptation to stress. Development 146:151894. http://doi.10.1242/dev.151894

27. Robinson MD, McCarthy DJ, Smyth GK (2010) EdgeR: a bioconductor package for differential expression analysis of digital gene expression data. Bioinformatics 26:139. http://doi.10.1093/bioinformatics/btp616

28. Sharma A, Wang JF, Xu DB, Tao SC, Chong SL, Yan DL, Li Z, Yuan HW, Zheng BS (2020) Melatonin regulates the functional components of photosynthesis, antioxidant system, gene expression, and metabolic pathways to induce drought resistance in grafted Carya cathayensis plants. Sci Total Environ 713:136675. http://doi.10.1016/j.scitotenv.2020.136675

29. Sheikh Mohammadi MH, Etemadi N, Arab MM, Aalifar M, Arab M, Pessarakli M (2017) Molecular and physiological responses of Iranian perennial ryegrass as affected by trinexapac ethyl, paclobutrazol and abscisic acid under drought stress. Plant Physiol Bioch 111:129-143. http://doi.10.1016/j.plaphy.2016.11.014

30. Shi H, Chan Z (2014) The cysteine2/histidine2-type transcription factor ZINC FINGER OF ARABIDOPSIS THALIANA 6-activated C-REPEAT-BINDING FACTOR pathway is essential for melatonin-mediated freezing stress resistance in Arabidopsis. J Pineal Res 57:185-191. http://doi.10.1111/jpi.12155

31. Shi HT, Jiang C, Ye TT, Tan DX, Reiter RJ, Zhang H, He YN, Li SY, He L, Chen W, Pan QH, Duan CQ, Chan ZL (2015) Comparative physiological, metabolomic, and transcriptomic analyses reveal 
mechanisms of improved abiotic stress resistance in bermudagrass [Cynodon dactylon (L). Pers.] by exogenous melatonin. J Exp Bot 66:681-694. http://doi.10.1093/jxb/eru373

32. Sun CL, Liu LJ, Wang LX, Li BH, Jin CW, Lin XY (2021) Melatonin: A master regulator of plant development and stress responses. J Integr Plant Bio/ 63:126-145. http://doi.10.1111/jipb.12993

33. Tang MJ, Xu L, Wang Y, Dong JH, Zhang XL, Wang K, Ying JL, Li C, Liu LW (2021) Melatonin-induced DNA demethylation of metal transporters and antioxidant genes alleviates lead stress in radish plants. Hortic Res 8:124. http://doi.10.1038/s41438-021-00561-8

34. Tian Y, Fan M, Qin ZX, Lv HJ, Wang MM, Zhang Z, Zhou WY, Zhao N, Li XH, Han C, Ding ZJ, Wang WF, Wang ZY, Bai MY (2018) Hydrogen peroxide positively regulates brassinosteroid signaling through oxidation of the BRASSINAZOLE-RESISTANT1 transcription factor. Nat Commun 9:1063. http://doi.10.1038/s41467-018-03463-x

35. Tiwari RK, Lal MK, Kumar R, Chourasia KN, Naga KC, Kumar D, Das SK, Zinta G (2021) Mechanistic insights on melatonin-mediated drought stress mitigation in plants. Physiol. Plantarum 172:12121226. http://doi.10.1111/ppl.13307

36. Wang L, Feng C, Zheng XD, Guo Y, Zhou FF, Shan DQ, Liu X, Kong J (2017) Plant mitochondria synthesize melatonin and enhance the tolerance of plants to drought stress. J Pineal Res 63: e12429. http://doi.10.1111/jpi.12429

37. Wang M, Dai WS, Du J, Ming RH, Dahro B, Liu JH (2019) ERF109 of trifoliate orange (Poncirus trifoliata (L.) Raf.) contributes to cold tolerance by directly regulating expression of Prx 1 involved in antioxidative process. Plant Biotechnol J 17:1316-1332. http:// doi.10.1111/pbi.13056

38. Wang YP, Reiter RJ, Chan ZL (2018) Phytomelatonin: a universal abiotic stress regulator. J Exp Bot 69:963-974. http://doi.10.1093/jxb/erx473

39. Wei J, Li DX, Zhang JR, Shan C, Rengel Z, Song ZB, Chen Q (2018) Phytomelatonin receptor PMTR1mediated signaling regulates stomatal closure in Arabidopsis thaliana. J Pineal Res 65:e12500. http://doi.10.1111/jpi.12500

40. Wei W, Li QT, Chu YN, Reirer RJ, Yu XM, Zhu DH, Zhang WK, Ma B, Lin Q, Zhang JS, Chen XE (2015) Melatonin enhances plant growth and abiotic stress tolerance in soybean plants. J Exp Bot 66:695707. http://doi.10.1093/jxb/eru392

41. Xia H, Ni ZY, Hu RP, Lin LJ, Deng HH, Wang J, Tang Y, Sun GC, Wang X, Li HX. Liao MG, Lv XL, Liang D (2020) Melatonin alleviates drought stress by a non-enzymatic and enzymatic antioxidative system in kiwifruit seedlings. Int J Mol Sci 21:852. http://doi.10.3390/ijms21030852

42. Xia XJ, Fang PP, Guo X, Qian XJ, Zhou J, Shi K, Zhou YH, Yu JQ (2018) Brassinosteroid-mediated apoplastic $\mathrm{H}_{2} \mathrm{O}_{2}$-glutaredoxin 12/14 cascade regulates antioxidant capacity in response to chilling in tomato. Plant Cell Environ 41:1052-1064. http://doi.10.1111/pce.13052

43. Xu LL, Xiang GQ, Sun QH, Ni Y, Jin ZX, Gao SW, Yao YX (2019) Melatonin enhances salt tolerance by promoting MYB108A-mediated ethylene biosynthesis in grapevines. Hortic Res 6:114. http://doi.10.1038/s41438-019-0197-4 
44. Yao JW, Ma Z, Ma YQ, Zhu Y, Lei MQ, Hao CY, Chen LY, Xu ZQ, Huang X (2021) Role of melatonin in UV-B signaling pathway and UV-B stress resistance in Arabidopsis thaliana. Plant Cell Environ 44:114-129. http://doi.10.1111/pce.13879

45. Ye H, Liu SZ, Tang BY, Chen JN, Xie ZL, Nolan TM (2017) RD26 mediates crosstalk between drought and brassinosteroid signalling pathways. Nat Commun 8: 14573. http://doi.10.1038/ncomms14573

46. Zhang J, Shi Y, Zhang XZ, Du HM, Xu B, Huang BR (2017) Melatonin suppression of heat-induced leaf senescence involves changes in abscisic acid and cytokinin biosynthesis and signaling pathways in perennial ryegrass (Lolium perenne L.). Environ Exp Bot 138:36-45. http://doi.10.1016/j.envexpbot.2017.02.012

47. Zheng X, Tan DX, Allan AC, Zuo BX, Zhao Y, Reiter RJ, Wang L, Wang Z, Guo Y, Zhou J, Shan D, Li Q, Han Z, Kong J (2017) Chloroplastic biosynthesis of melatonin and its involvement in protection of plants from salt stress. Sci Rep 7: 41236. http://doi.10.1038/srep41236

\section{Figures}


(a)

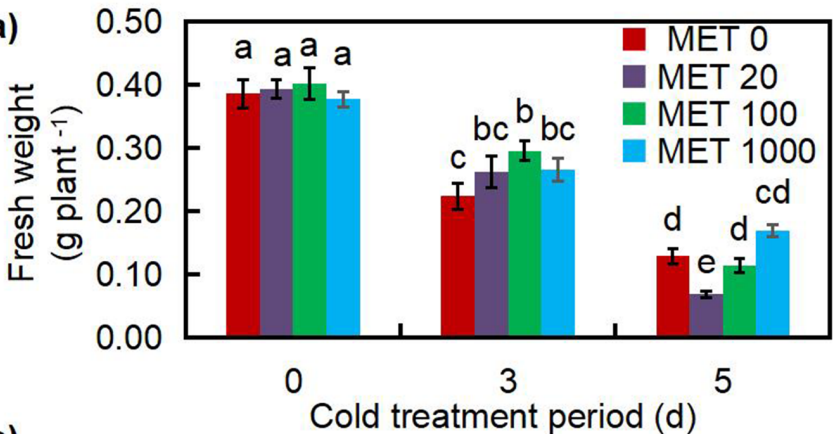

(c)

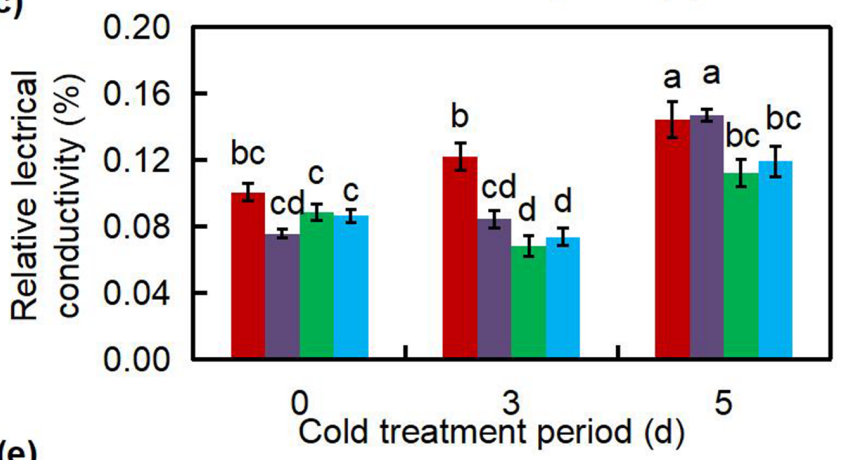

(e)

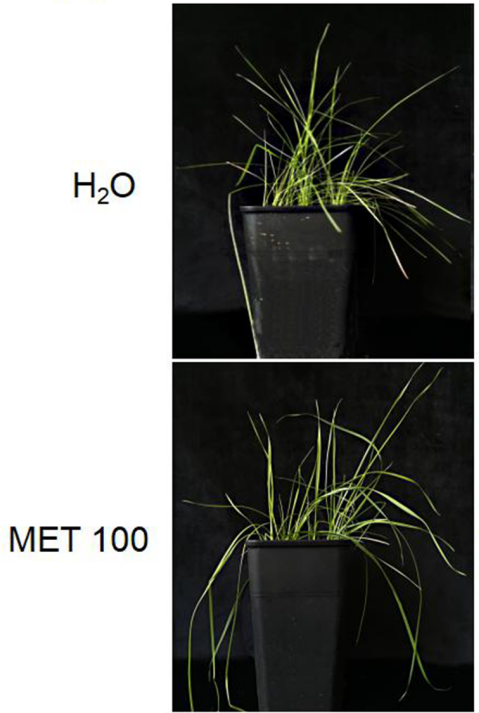

CK

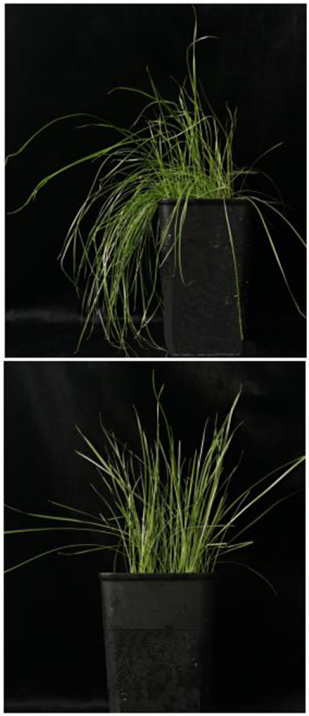

$3 d$
$5 d$
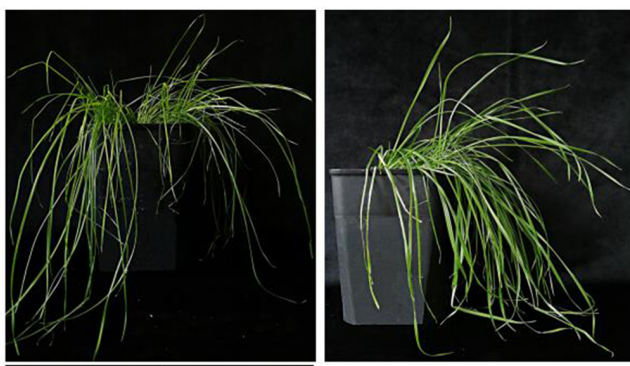

5

(d)

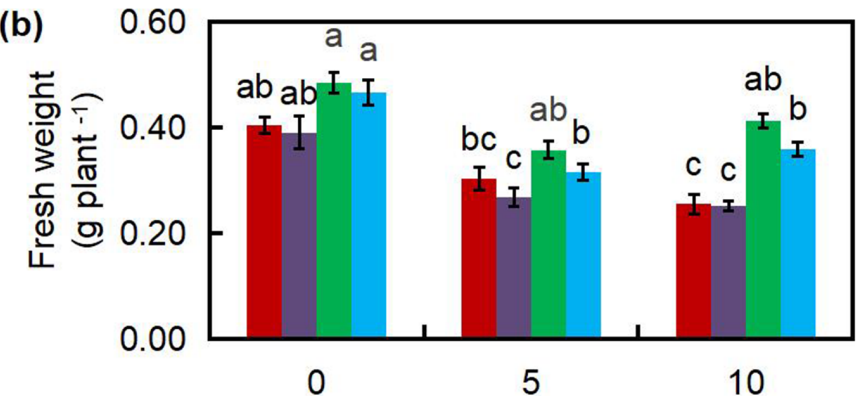

Drought treatment period (d)
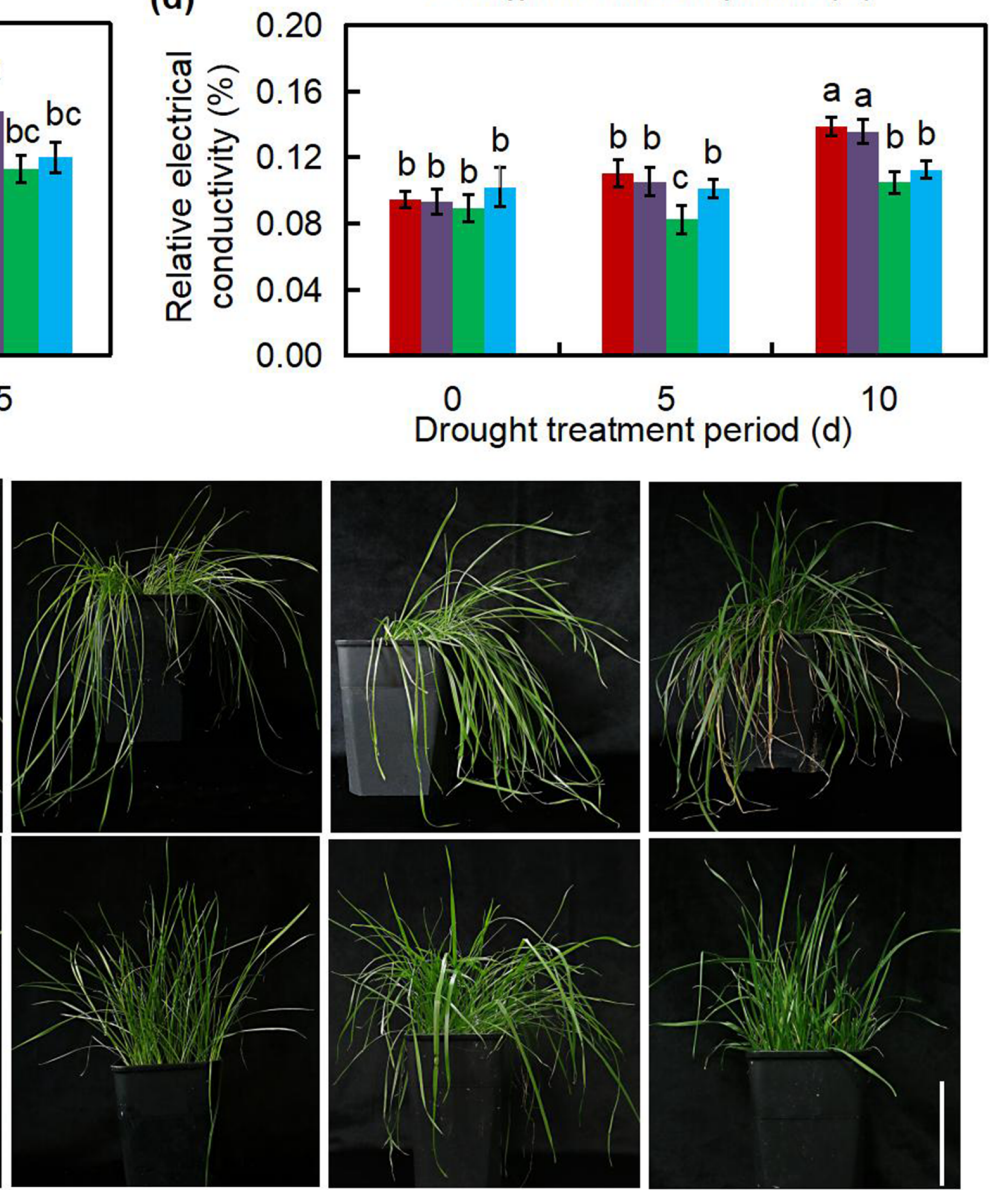

$10 \mathrm{~d}$

Drought

\section{Figure 1}

Melatonin mediates cold and drought tolerance in a concentration dependent manner in perennial ryegrass. $(a, b)$ Changes in fresh weight in different concentration of $\operatorname{MET}(0,20,100,1,000 \mu M)$ treatment exposed to cold and drought stress. (c, d) Changes in relative electrolyte leakage in the presence or absence of MET under cold and drought stress. (e) Phenotpype response in the presence 100 $\mu \mathrm{M}$ MET under cold and drought stress for the mentioned peroid. Scale bars, $3 \mathrm{~cm}$. The 4-week-old plants 
were sprayed with MET or water for $7 \mathrm{~d}$, and then subjected to cold and drought stress for the mentioned peroid. Non-treated seedlings were used as the control

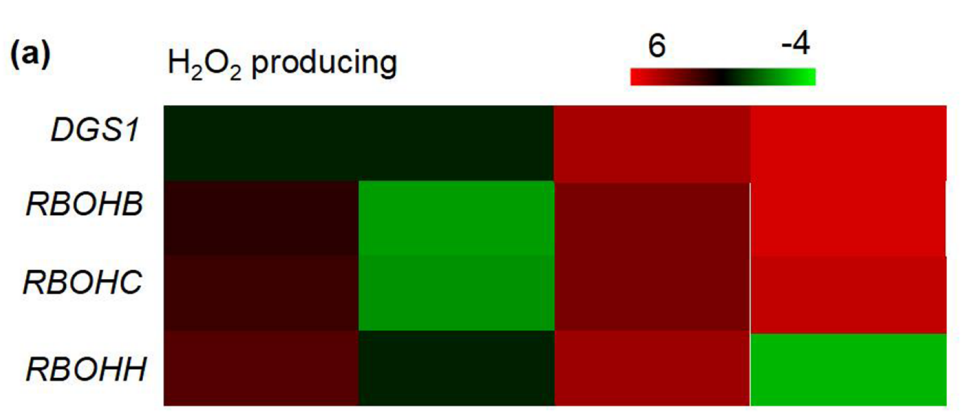

(c)

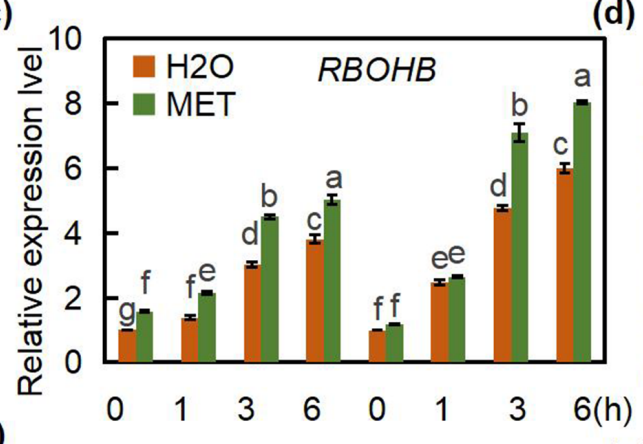

(f)

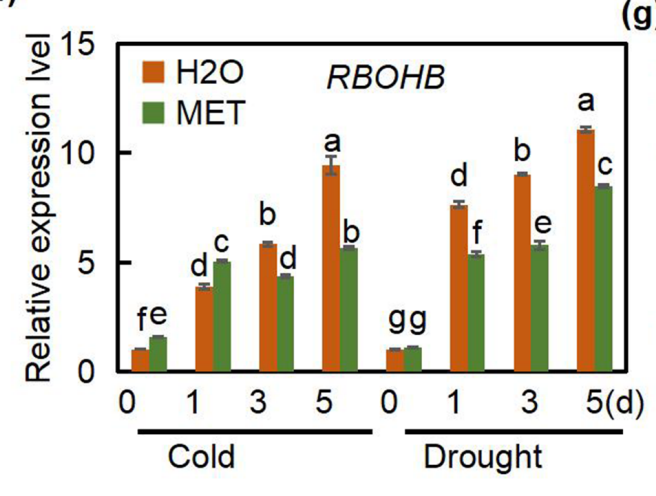

(d)

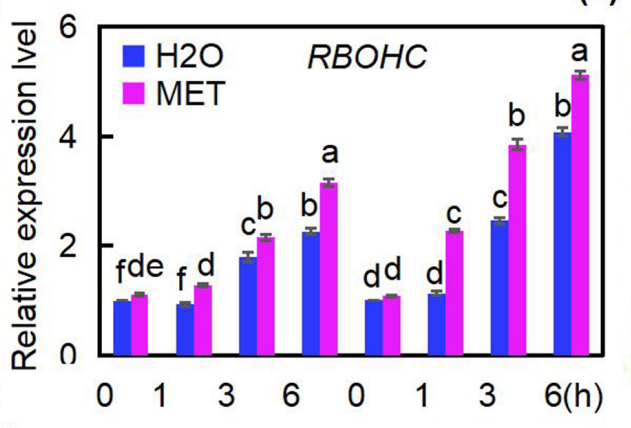

(e) (b)

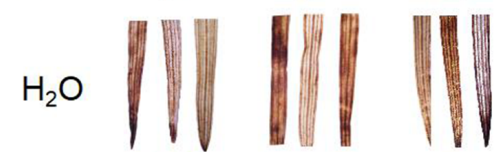

MET

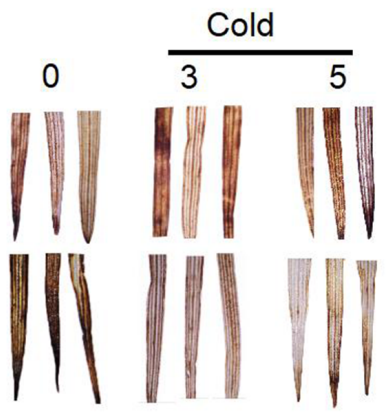

$\frac{\text { Drought }}{5 \quad 10} d$
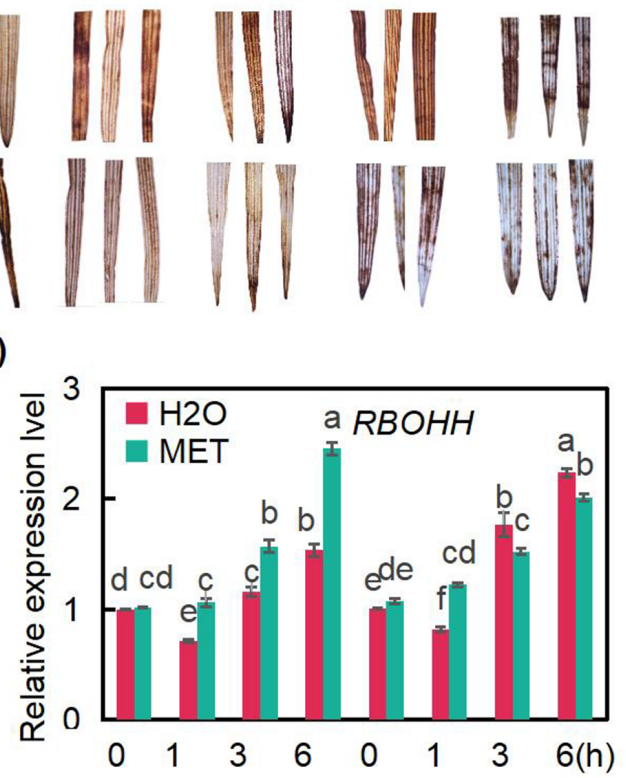

(h)
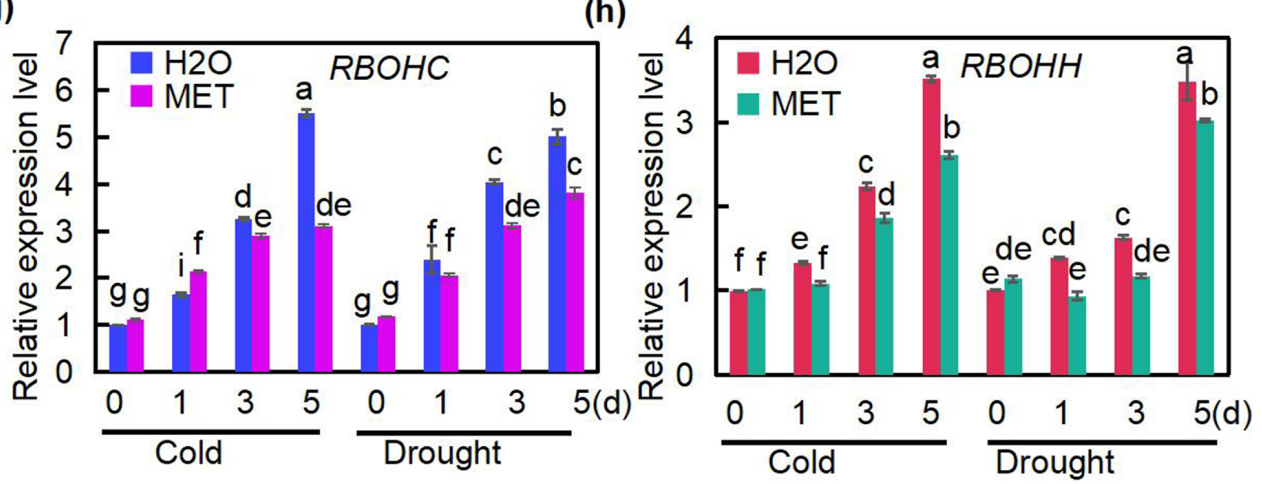

Figure 2

Melatonin has contrasting effects of $\mathrm{H} 2 \mathrm{O} 2$ producing under short- and long-term cold and drought stress. (a) Heat-map of the RNA-Seq expression profiles of $\mathrm{H} 2 \mathrm{O} 2$ biosynthesis genes. (b) DAB staining for $\mathrm{H} 2 \mathrm{O} 2$ production in the MET-treated or non-treated plants after $5 \mathrm{~d}$ of cold and $10 \mathrm{~d}$ of drought treatments. (c-h) RT-qPCR analysis of H2O2 biosynthesis genes. Three biological replicates were performed. Samples were 

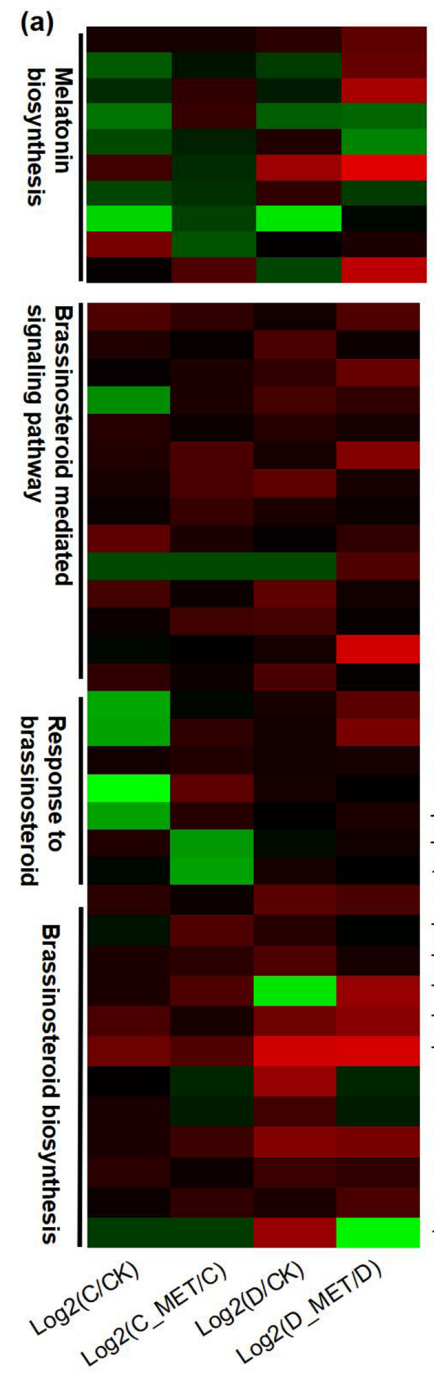

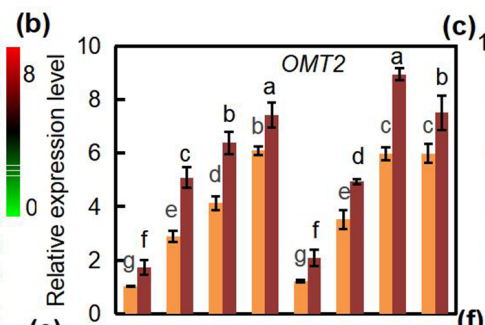

(c)

TRINITY_DN36055 c2 g2 TRINITY_DN28152_CO_g4 TRINITY_DN39870_c0_g5 TRINITY_DN31823_c0_g2 TRINITY-DN39870 CO g1 TRINITY_DN27001_c2_g8 TRINITY_DN42680_c1_g2 TRINITY_DN33542_c0_g3 TRINITY DN39870 c0 g3 TRINITY_DN25938_c1_g3

TRINITY_DN29451_c2_g4 TRINITY_DN35603_c0_g4 TRINITY_DN43321_c1_g2 TRINITY DN44133 c0 g2 TRINITY_DN29416_c1_g1 TRINITY_DN25933_c2_g6 TRINITY DN29451 c2 g7 TRINITY_DN42280_c0_g2 TRINITY_DN42300_C3_g1 TRINITY_DN34298_c1_g4 TRINITY_DN44248_c0_g1 TRINITY_DN28325_c0_g1 TRINITY_DN19209_c0_g1 TRINITY_DN29727_c1_g1 TRINITY_DN40033_c0_g3 TRINITY_DN32547_c0_g5 TRINITY_DN43175_c0_g1 TRINITY_DN31576 c0 g2 TRINITY_DN37697_c0_g3 TRINITY_DN31869_c0_g1 TRINITY DN30788 c0 g1 TRINITY_DN39688 c0 g7 TRINITY_DN33606_c0_g1 TRINITY_DN33606_c0_g2 TRINITY_DN43313_c0_g2 TRINITY_DN43313_c0_g5 TRINITY DN29413 c0 g1 TRINITY_DN23508_c0_g1 TRINITY_DN26756_c0_g1 TRINITY DN32175 c0 g5 TRINITY_DN37595_c0_g3 TRINITY_DN42022_c0_g2 TRINITY_DN35701_c0_g5 (e)
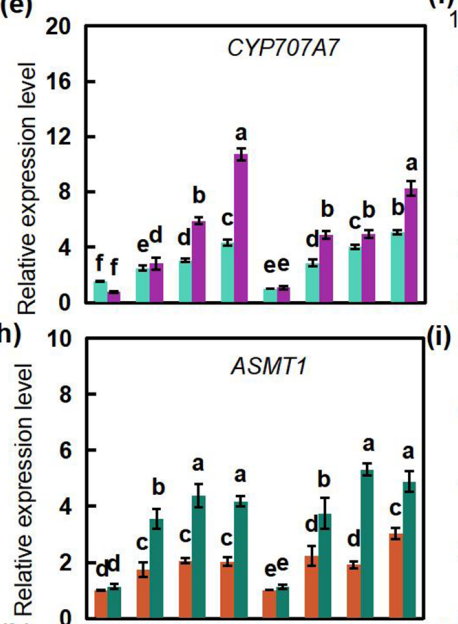

(k)

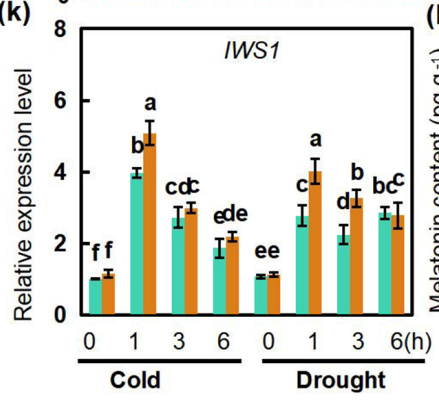

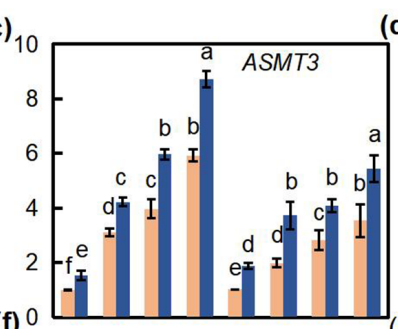

(d)

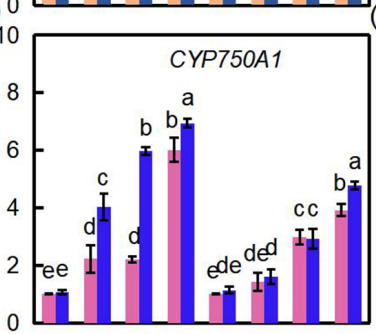

(g) 0

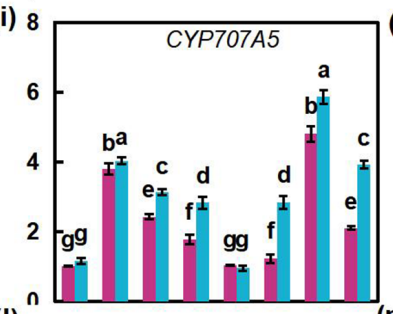

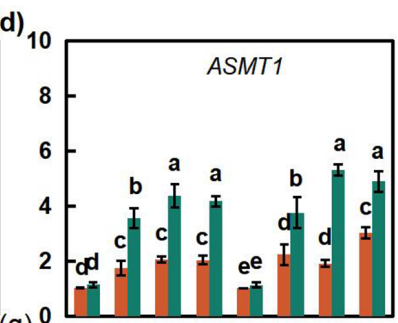

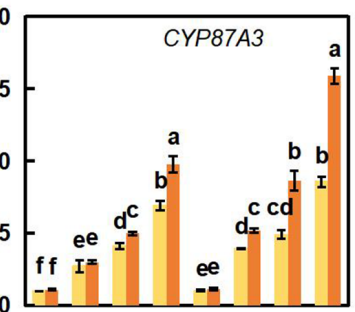

(I) 200
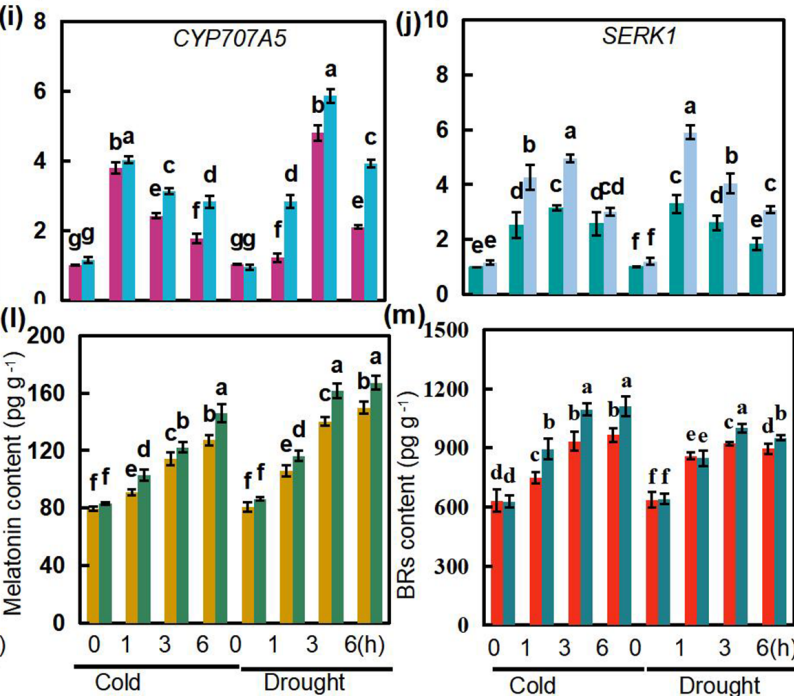

(m)

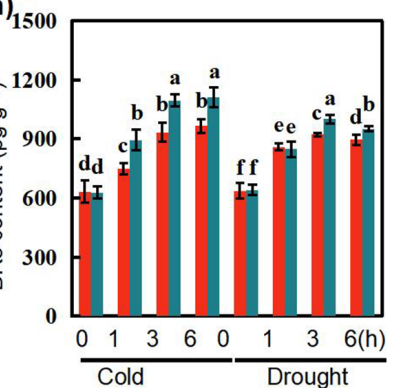

\section{Figure 3}

Both cold and drought stress induce melatonin and brassinosteroids biosynthesis in perennial ryegrass.

(a) Heatmaps of the expresion of biosynthesis or signaling-related genes of MET and BRs. C, Cold. D, Drought, C_MET, combined with MET and cold treatment, D_MET, combined with MET and drought treatment. (b-d) Time course of MET biosynthesis genes transcription induced by $100 \mu \mathrm{M}$ MET exposed 
to cold and drought stress via RT-qPCR analysis. (e-k) Changes in relative transcription level of genes involved in BRs biosynthesis and signaling transduction. $(\mathrm{i}, \mathrm{m})$ Changes in endogenous MET and BRs levels. In figures b-m, the left and right columns represent water and MET treatment, respectively. Three biological replicates were performed. Samples were collected from three independent experiments. Bars indicate means $\pm S D$. The different lowercase letters represent significant differences at $P<0.05$ (Duncan's test)

(a)

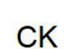
Cold Cold +MET Cold+BR Cold + MET $+P P Z$ Drought

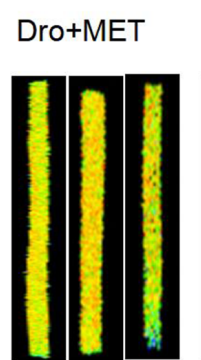
Dro+BR Dro+MET

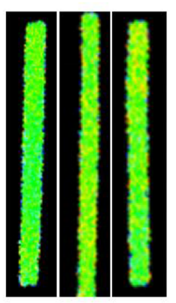

(b)
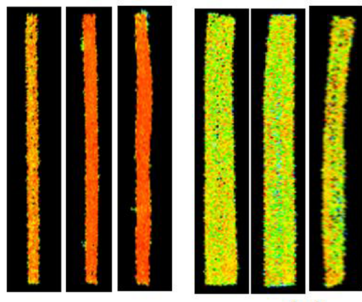

(c)

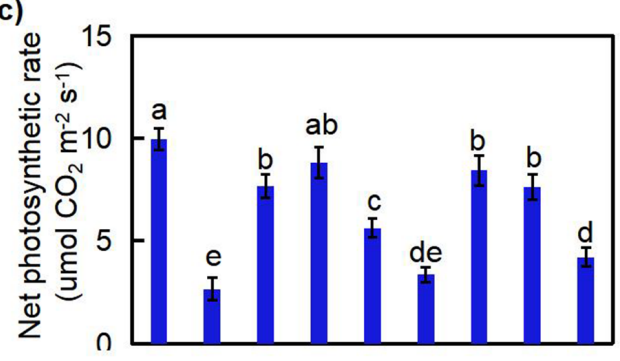

(e)
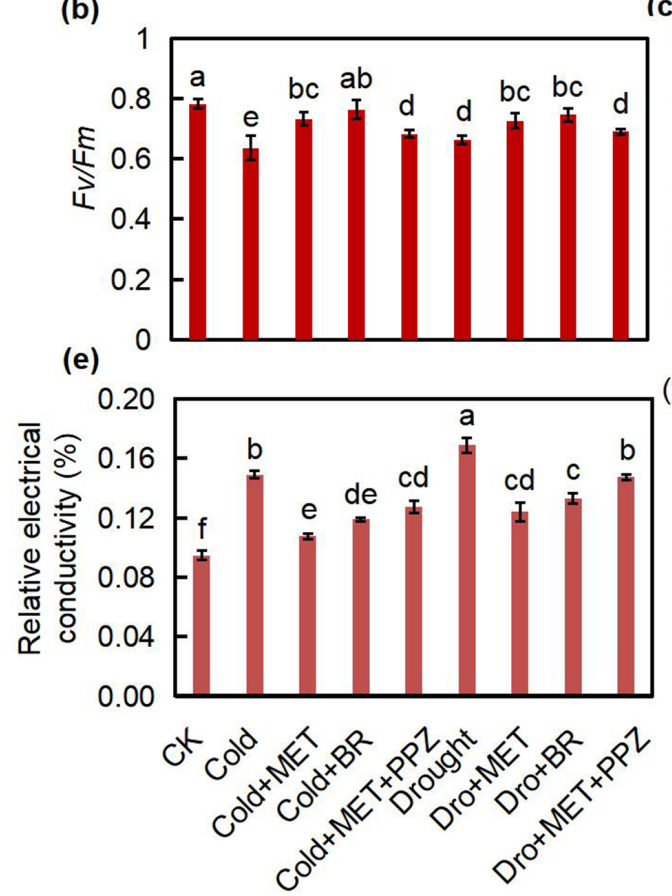
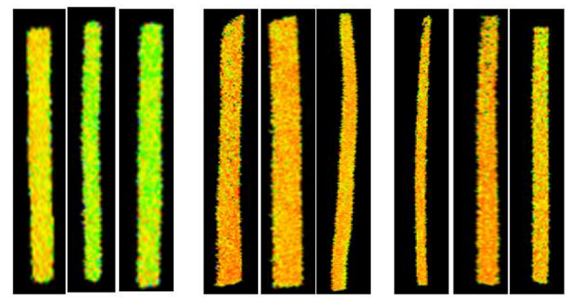

(d)

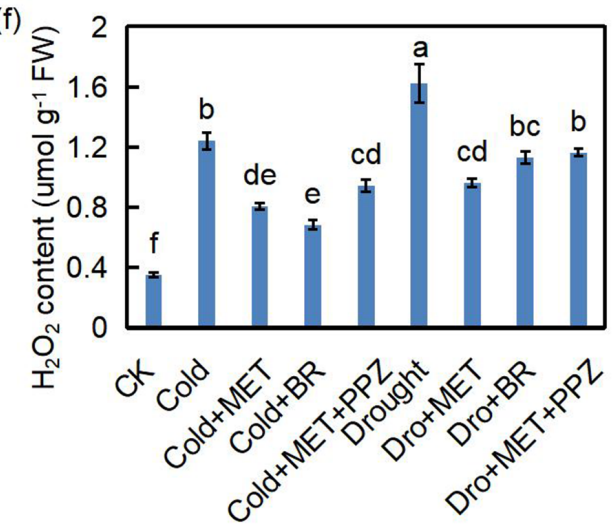

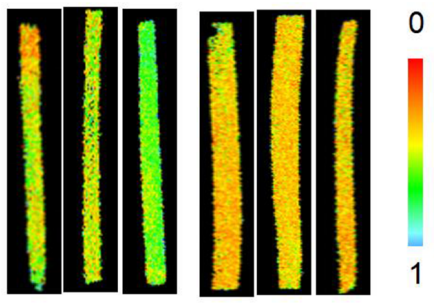

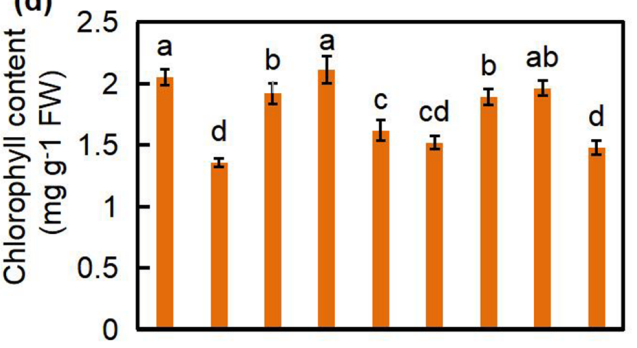

\section{Figure 4}


Melatonin positively regulates cold and drought tolerance of perennial ryegrass partially through brassinosteroids. (a) Images of maximum photochemical efficiency of PSII (Fv/Fm) in the presense of MET, BRs, and BRs synthesis inhibitor PPZ or water exposed to $5 \mathrm{~d}$ of cold and $10 \mathrm{~d}$ of drought stress. (b) Comparison of the average values of Fv/Fm. (c) Changes in net photosynthetic rate (Pn), and (d) chlorophyll (Chl) content. (e) Relative electrolyte leakage in MET, BR and MET plus PPZ-treated seedlings exposed to either cold or drought stresses. (f) Quantification of $\mathrm{H} 2 \mathrm{O} 2$ content in the leaves of perennial ryegrass seedlings. Three biological replicates were performed. Samples were collected from three independent experiments. Bars indicate means \pm SD. The different lowercase letters represent significant differences at $P<0.05$ (Duncan's test) 
(a)

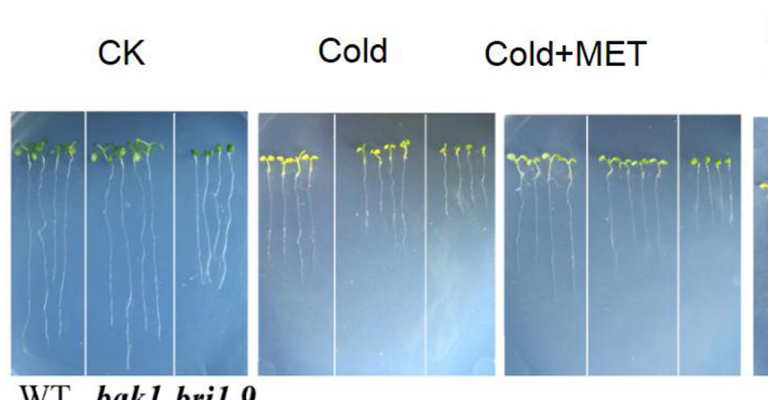

WT bakl bril.9

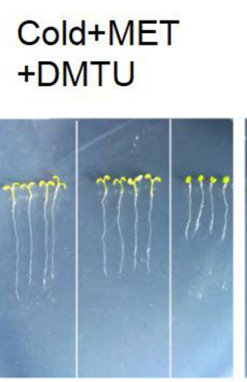

Drought

Dro+MET

Dro+MET

+ DMTU
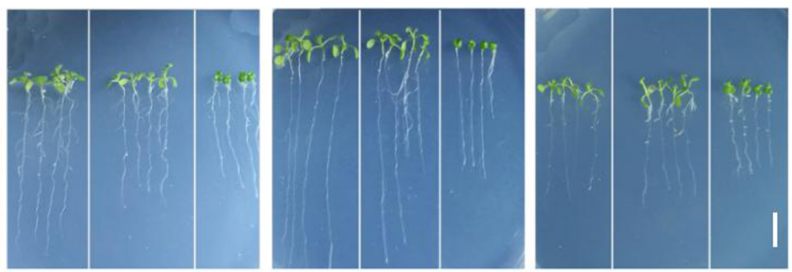

(b)

CK

Cold

Cold+MET

Drought

Dro+MET
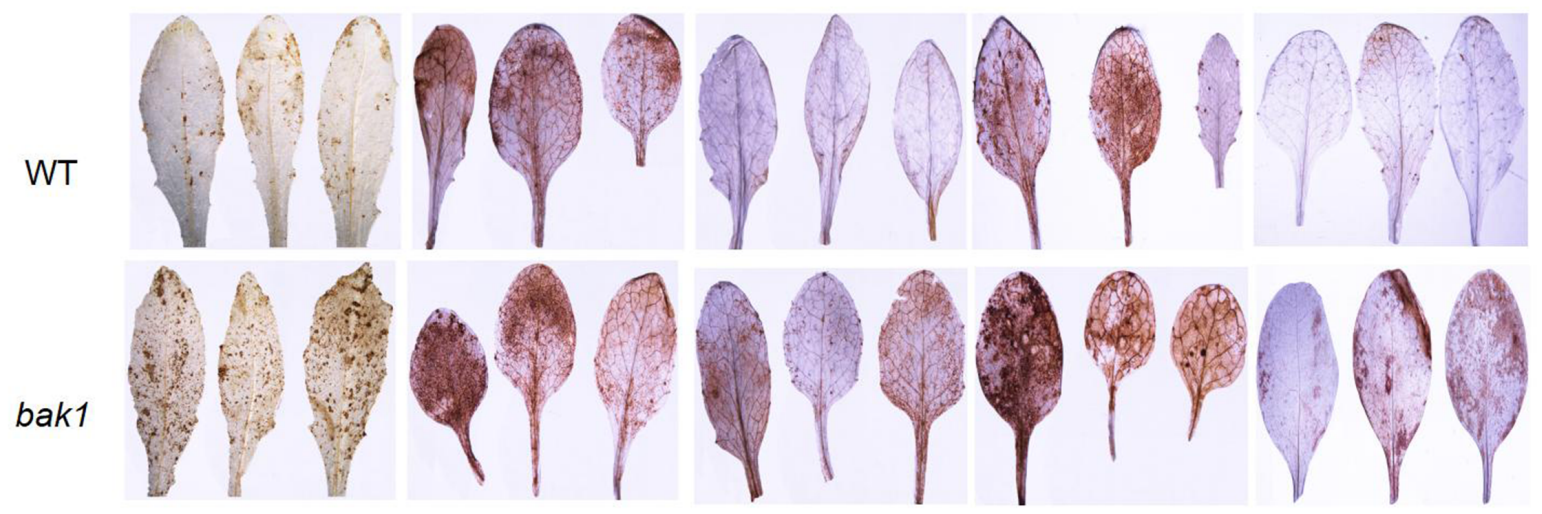

\section{Figure 5}

A crosstalk between BRs and $\mathrm{H} 2 \mathrm{O} 2$ signaling involved in the melatonin -mediated cold and drought tolerance. (a) Phenotype of seven-day old wild type (WT), bri1-9, and bak1 mutants grown on standard $1 / 2$ MS medium pretreated with $50 \mu \mathrm{M}$ MET and $0.5 \mathrm{mM} \mathrm{H} 2 \mathrm{O} 2$ scavenger DMTU expose to cold and drought stress. Bar, $1 \mathrm{~mm}$. (b) Histochemical staining of H2O2 for the MET and/or DMTU-treated leaves from WT and bak1 mutants 


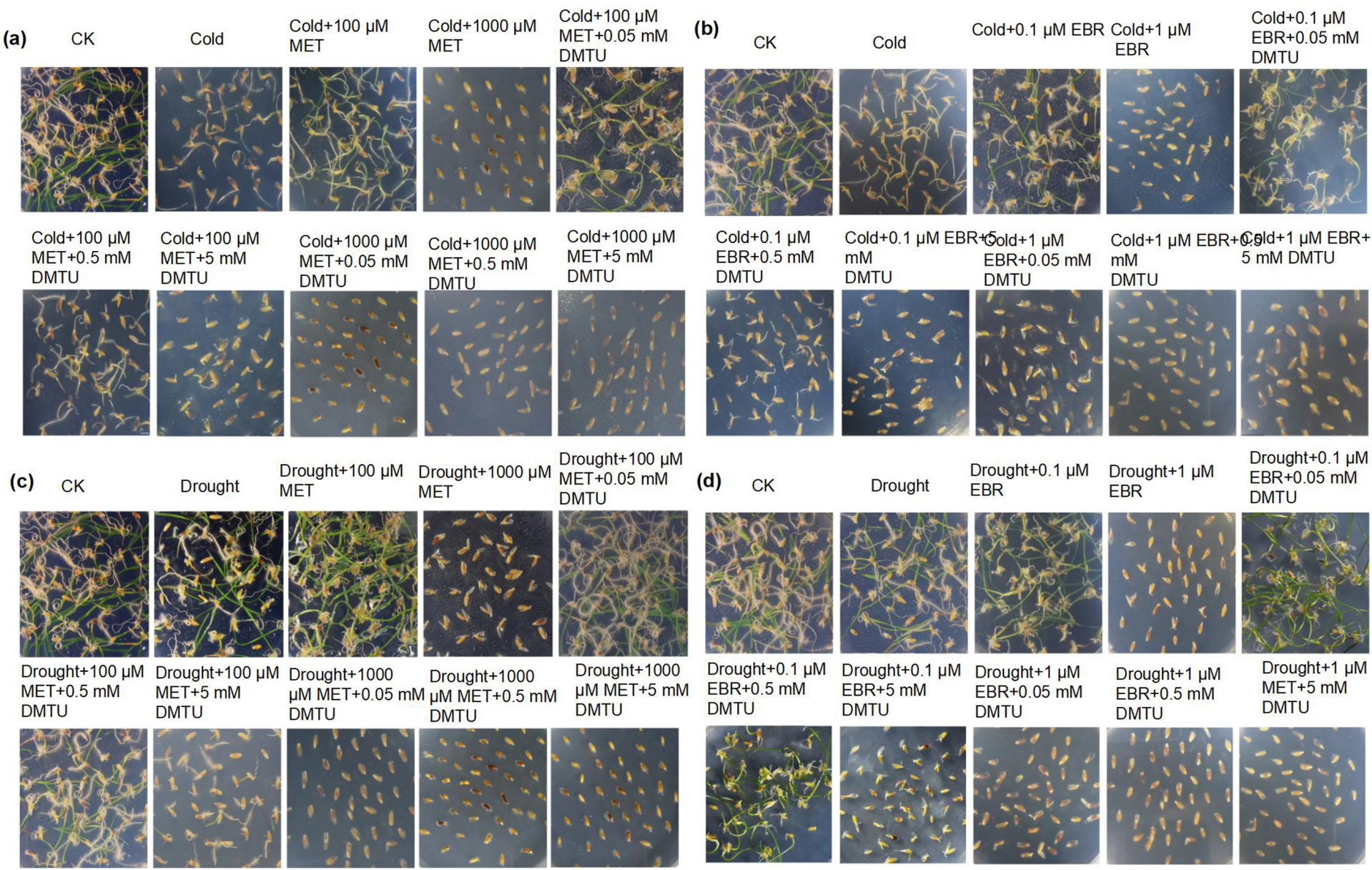

\section{Figure 6}

$\mathrm{H} 2 \mathrm{O} 2$ is required for melatonin and brassinosteroids increase seed germination rate under cold and drought stress. Seeds were germinated on standard 1/2 MS medium exposed to $40 \mathrm{C}(\mathrm{a}, \mathrm{b})$ and $250 \mathrm{mM}$ mannitol (c, d) with or without $100 \mu \mathrm{M}$ MET, 1,000 $\mu \mathrm{M}$ MET, $0.1 \mu \mathrm{M}$ BRs (24-epibrassinolide, a BRs donor) , $1 \mu \mathrm{M}$ BRs , 100 or $1,000 \mu \mathrm{M}$ MET plus $0.05,0.5$ or $5 \mathrm{mM}$ DMTU (dimethylthiourea, $\mathrm{H} 2 \mathrm{O} 2$ scavenger ), and 0.1 or $1 \mu \mathrm{M}$ BRs plus $0.05,0.5$ or $5 \mathrm{mM}$ DMTU treatment 
(a)

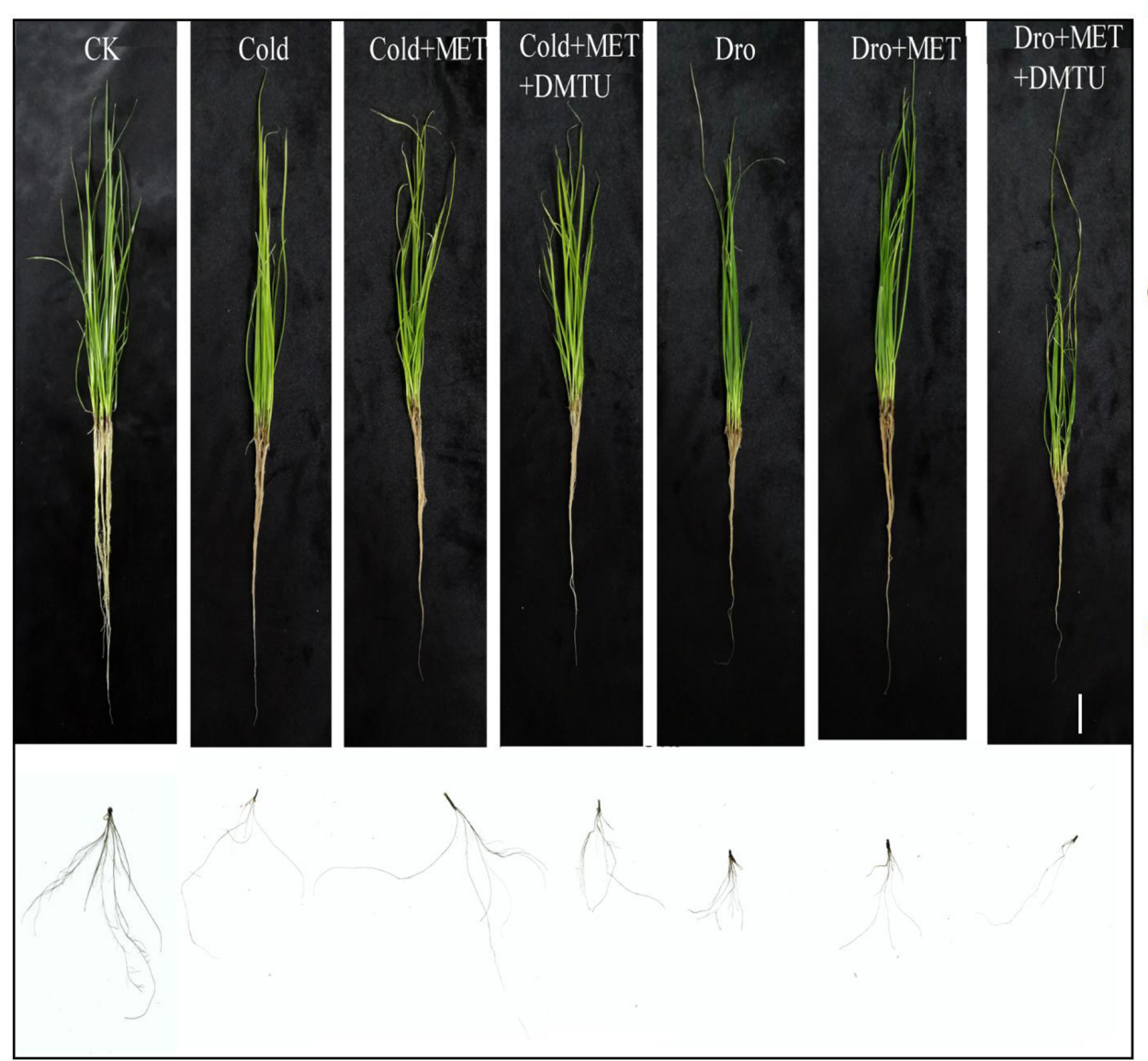

(b)

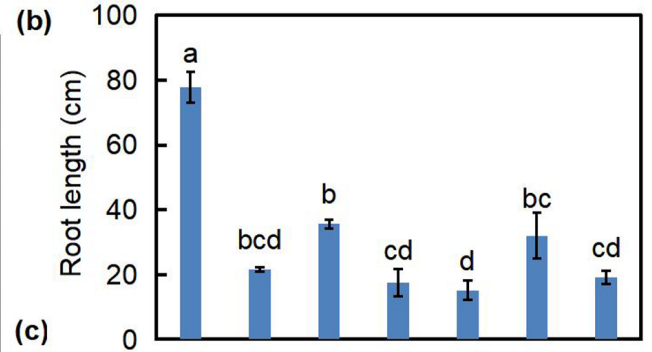

(c)

(d)
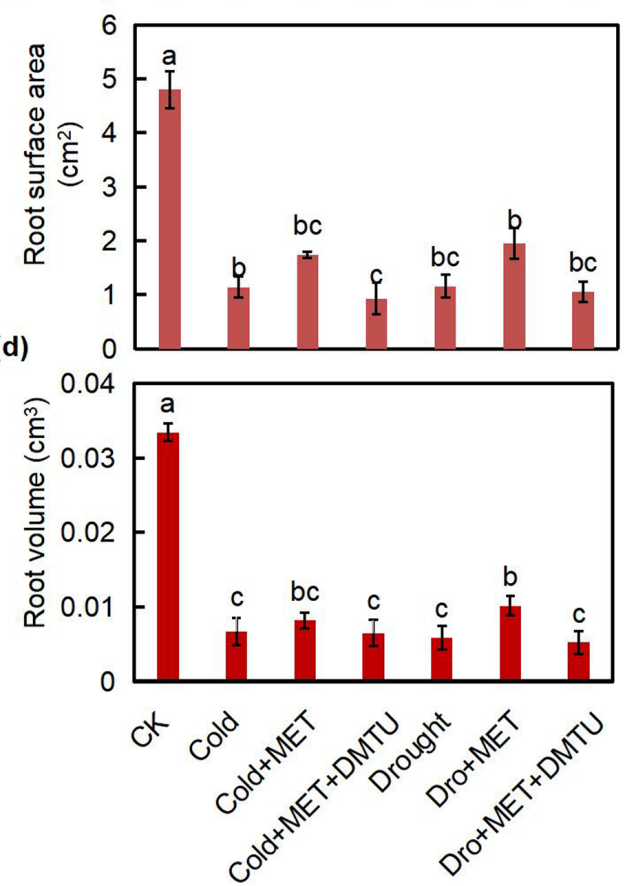

Figure 7

Melatonin and brassinosteroids improved seedlings growth closely related to $\mathrm{H} 2 \mathrm{O} 2$ levels under cold and drought stress. (a) The root phenotpype seedlings under $40 \mathrm{C}$ or $250 \mathrm{mM}$ mannitol treatment for $7 \mathrm{~d}$. Bar, $1 \mathrm{~cm}$. (b-d) Measurement of root length, surface area and volume. Three biological replicates were performed. Samples were collected from three independent experiments. Bars indicate means \pm SD. The different lowercase letters represent significant differences at $\mathrm{P}<0.05$ (Duncan's test) 


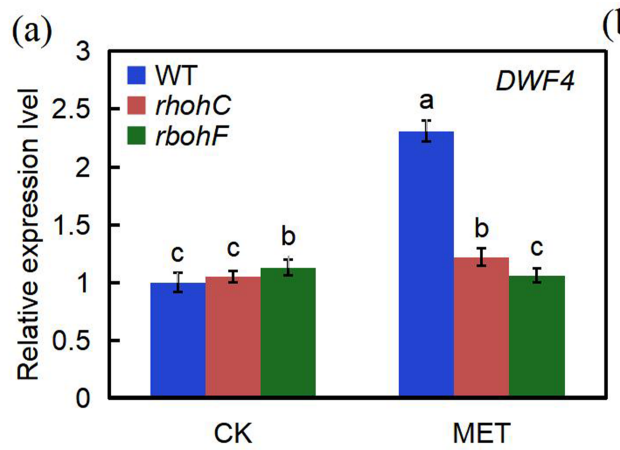

(b)
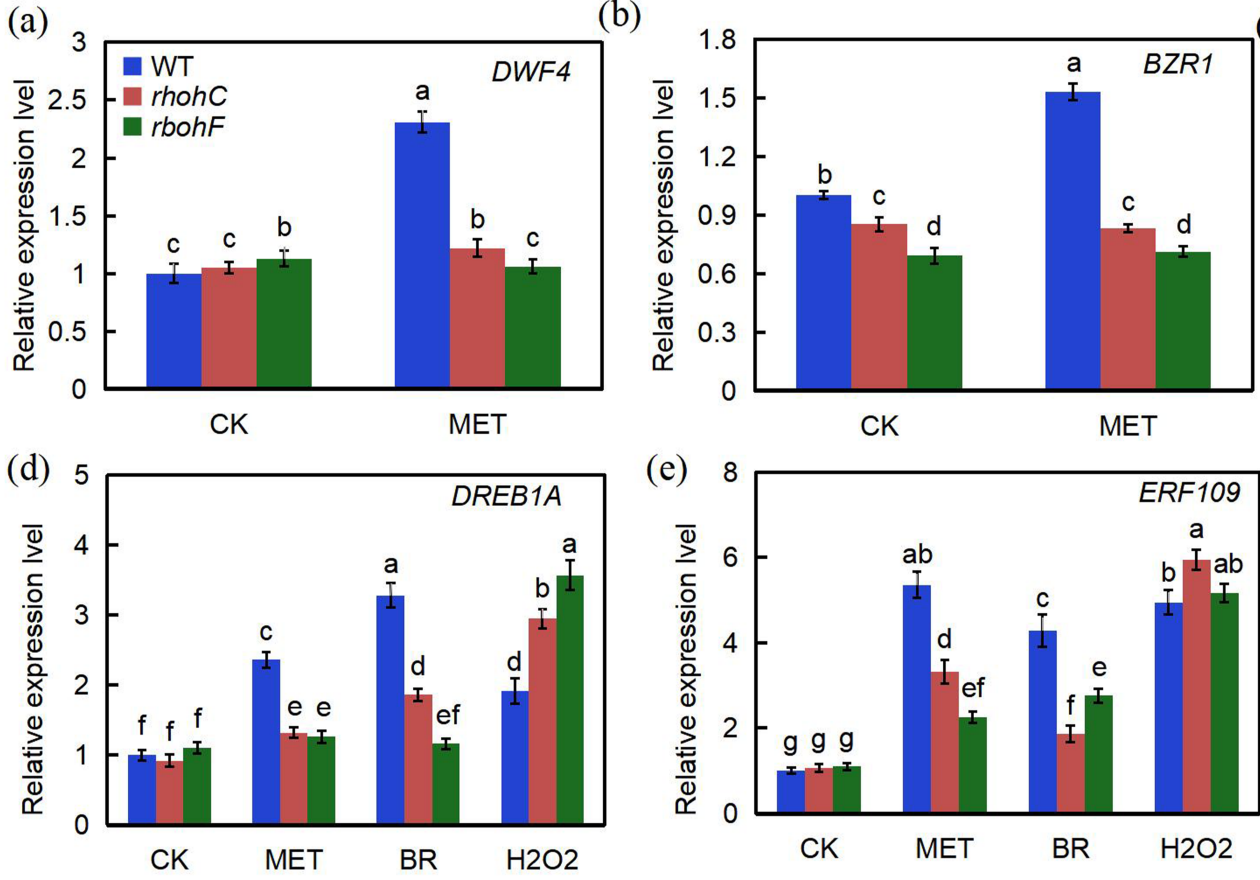

(e)

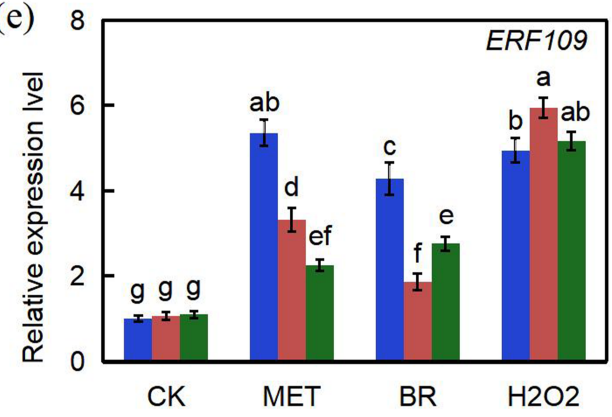

(c)

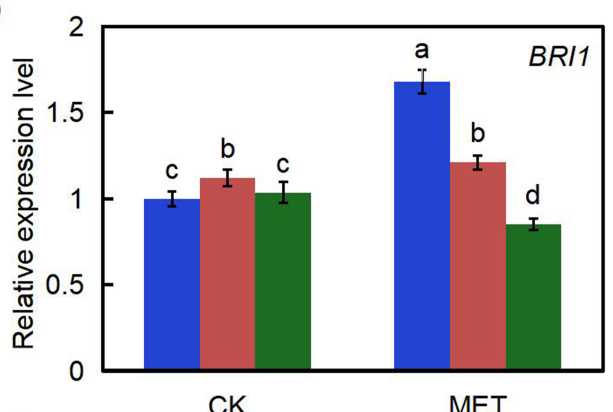

(f)

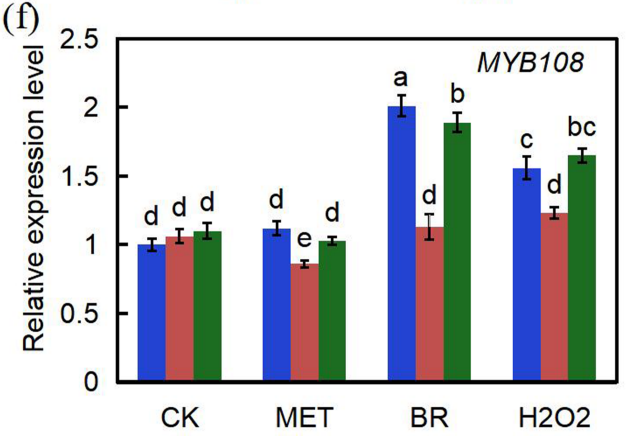

\section{Figure 8}

Involvement of $\mathrm{RBOH}$-dependent $\mathrm{H} 2 \mathrm{O} 2$ production in melatonin and brassinosteroids-induced stress responsive process. (a-c) Changes in the expression of DWF4, BZR1 (BRASSI NAZOLE-RESISTANT 1) and BRI1 (BRASSINOSTEROID-INSENSITIVE 1) genes in the WT and rbohC and rbohF mutants in the presence of $50 \mu \mathrm{M}$ MET or water under normal conditions. (d-f) Expression of stress-responsive genes in the leaves of WT and rbohC and rbohF mutants ptre-treated with water or $50 \mu \mathrm{M}$ MEL, $1 \mu \mathrm{M} \mathrm{EBL}$, and $100 \mu \mathrm{M} \mathrm{H} 2 \mathrm{O} 2$ under normal conditions. The $14 \mathrm{~d}$-old soil-grown rbohC and rbohF seedlings were sprayed for MET for 12 h. Three biological replicates were performed. Samples were collected from three independent experiments. Bars indicate means \pm SD. The different lowercase letters represent significant differences at $P<0.05$ (Duncan's test) 


\section{Exogenous melatonin}
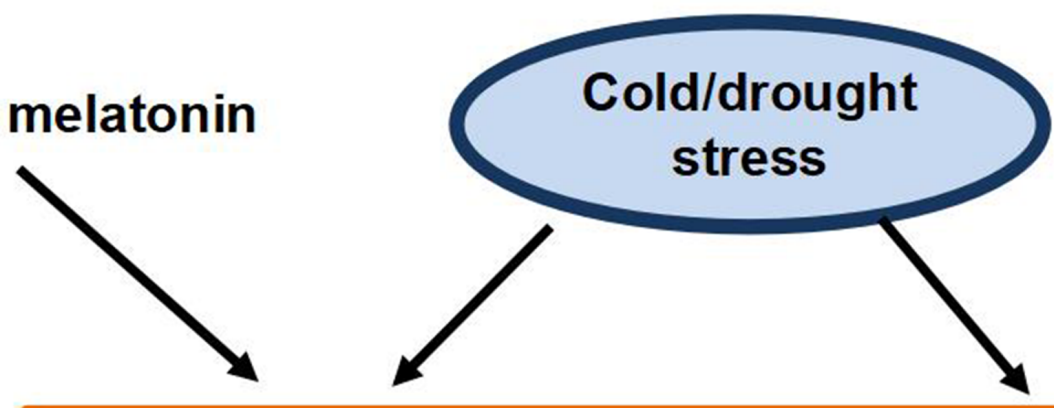

\section{Melatonin $\longrightarrow$ Brassinosteroid}
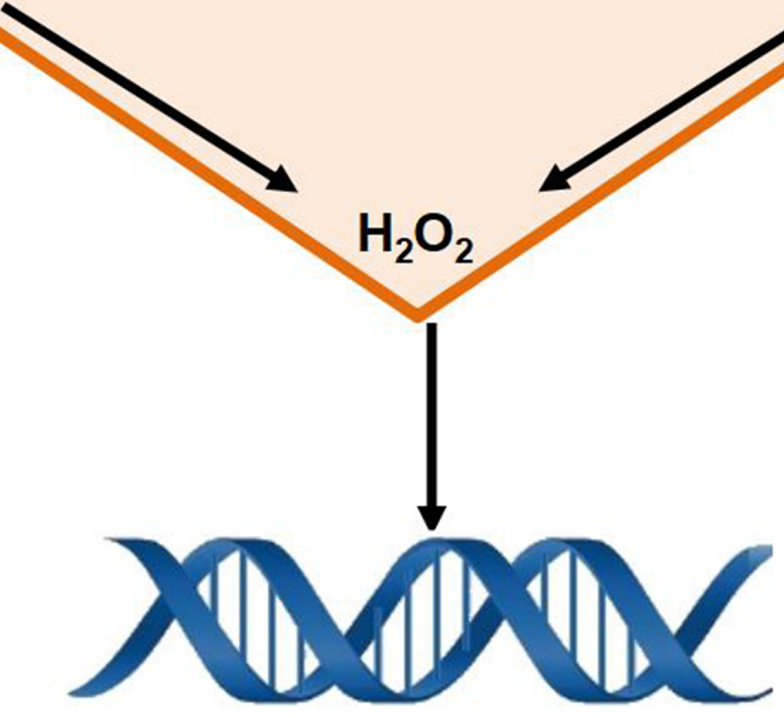

\section{CBF/DREB1A; ERFs;WRKYs;MYBs ...}

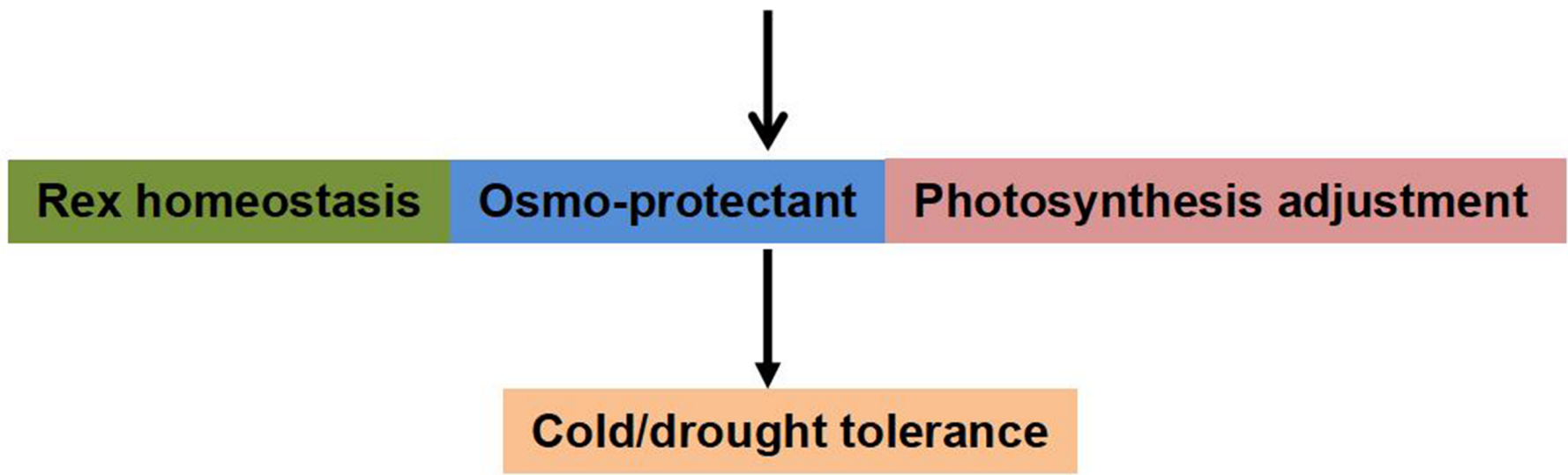

Figure 9

Proposed model to explain melatonin-BRs-H2O2 signaling regulates cold and drought tolerance in perennial ryegrass. Melatonin increases the levels of endogenous melatonin and the induction of BRs biosynthesis and signaling pathway in the plants exposed to initial phases of stress. In this process, the NADPH oxidase-dependent $\mathrm{H} 2 \mathrm{O} 2$ signaling might acts as a downstream component, and this signaling network further activates relevant transcription factors and stress-related genes 


\section{Supplementary Files}

This is a list of supplementary files associated with this preprint. Click to download.

- Supplementaryfigures.pdf

- Tables1.xls

- Tables2.xlsx

- Tables3.xls 